\title{
Metal-based Antimicrobial agents: Synthesis, Characterization and Biological studies of Mannich base derivatives of Benzimidazole and their Metal complexes
}

\author{
Misbah ur Rehman ${ }^{1}$, Muhammad Imran ${ }^{2, ~ *, ~ M u h a m m a d ~ A r i f ~}{ }^{2}$, Muhammad Farooq $^{3}$ \\ ${ }^{1}$ Institute of Chemical Sciences, Gomal University, D. I. Khan, KPK, Pakistan \\ ${ }^{2}$ Institute of Chemical Sciences, Bahauddin Zakariya University, Multan, Pakistan \\ ${ }^{3}$ Department of Chemistry, Govt. College, Gujranwala, Pakistan
}

\section{Email address:}

imran345@hotmail.com (M. Imran)

\section{To cite this article:}

Misbah ur Rehman, Muhammad Imran, Muhammad Arif, Muhammad Farooq. Metal-based Antimicrobial Agents: Synthesis, Characterization and Biological Studies of Mannich Base Derivatives of Benzimidazole and Their Metal Complexes. Science Journal of Chemistry. Vol. 1, No. 5, 2013, pp. 56-66. doi: 10.11648/j.sjc.20130105.12

\begin{abstract}
Novel Mannich base derivatives of Benzimidazole were prepared through the condensation reaction of benzimidazole derivative with formaldehyde and primary and/ secondary amine. Zinc(II), copper(II), nickel(II) and cobalt(II) complexes of Mannich bases have also been synthesized. All the compounds were fully characterized by, elemental analyses, magnetic moment determination, molar conductivity measurement, thermogravimetric analysis, spectral and analytical data. Experimental results showed that metal complexes act as bi-dentate ligands towards divalent metal ions via azomethine-N and deprotonated-O while suggesting an octahedral geometry. All the compounds were screened for in-vitro antibacterial and antifungal activity against various bacterial and fungal strains. Almost all the compounds showed good potent activity against microorganisms. It was also seen that compounds with complexed form were more active as compared to un-complexed form. The prepared compounds were also screened for their cytotoxicity and results showed that only $\mathrm{Ni}$ (II) complexes exhibit some cytotoxicity while all other compounds were almost inactive.
\end{abstract}

Keywords: Mannich Bases, Benzimidazole, Metal Complexes, Biological Activity, Cytotoxicity

\section{Introduction}

The benzimidazoles contain a phenyl ring fused to an imidazole ring. Benzimidazole and their derivatives have diverse applications in coordination chemistry, photophysics, photochemistry and bioinorganic chemistry.[1-4] Over the past few decades, Mannich base reactions of benzimidazole have been the guiding tent for the synthetic chemists because of their widespread pharmaceutical importance i.e. antibacterial, anthelmintic[5], antifungal[6], anti-inflamatory[7], antiviral[8] and analgesic[9] properties. The compounds with azomethine group in its structure are known as Schiff bases, which are synthesized by the condensation reaction of primary amines and active carbonyl groups.[10] Schiff bases of benzimidazole have been reported with remarkable antibacterial[11], antimicrobial[12] and antiproliferative[13] activities. In addition to their biological importance, benzimidazoles form stable complexes with various transition metals.[14] Transition metal complexes of 2-substituted benzimidazole and benzimidazole-based mixed ligands have been reported with mono-, bi- and tri dentate coordination behavior.[15-19]

The worthwhile biological activities of Mannich and Schiff bases have been guiding for the synthesis of novel Mannich and Schiff bases in a single molecule. The main objective of present communication is to provide a comprehensive account of N-Mannich type bases of benzimidazole, their chelating behavior and to highlight their potential in evolving better antimicrobial drugs. A total of 6 Mannich and Schiff bases and 24 metal(II) complexes have been prepared in this study and well characterized by their physical, spectral and analytical data. The synthesized compounds were further evaluated for their antimicrobial properties against various pathogens using MIC method. 


\section{Experimental}

\subsection{General Manipulations}

All the reagents and solvents were purchased from Sigma-Aldrich and they were used as received. Reactions were monitored by thin layer chromatography (plates coated with $0.2 \mathrm{~mm}$ Merck 60 F254 silica gel) and were visualized by UV irradiation $(254 \mathrm{~nm})$. Elemental analyses were carried out with a LECO-CHNS-9320 model. ${ }^{1} \mathrm{H}$ and ${ }^{13} \mathrm{C}-\mathrm{NMR}$ spectra of compounds were recorded with a Bruker Spectrospin Avance DPX-400 using TMS as internal standard and d6 DMSO as solvent. Infrared spectra of compounds were recorded on a Philips Analytical PU 9800 FTIR spectrophotometer. The melting points of compounds were determined with a Gallenkamp melting point apparatus. UV/visible absorption spectra were recorded using a Shimadzu UV-1700 spectrophotometer at room temperature. Conductance was recorded by pre-calibrated cyber scan 500 conductivity meter. Electron impact mass spectra (EIMS) were recorded on a JEOL MS Route instrument. Thermogravimetric analysis (TGA) was carried out under constant nitrogen flow at a heating rate of $15^{\circ} \mathrm{C}$ min-1, using a Mettler Toledo TGA/SDTA 851 balance. The heating scans were performed on 3-5 $\mathrm{mg}$ of sample, in the temperature range $25-900{ }^{\circ} \mathrm{C}$. In vitro antibacterial, antifungal and cytotoxic properties were studied at HEJ Research Institute of Chemistry, International Center for Chemical Sciences, University of Karachi, Pakistan.

\subsection{Synthesis of Schiff base (Scheme 1)}

Schiff base of 2-amino(methyl)benzimidazole with salicyldehyde was prepared according to the method reported in literature[20].

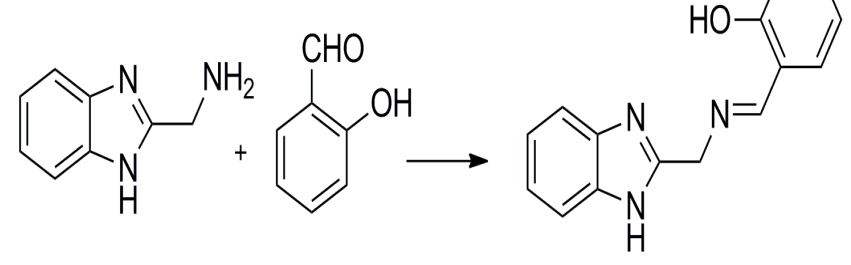

Scheme 1. Synthesis of Schiff base.

\subsection{Synthesis of Mannich bases (Scheme 2)}

To a solution of synthesized Schiff base $(0.05$ mole $)$ in $30 \mathrm{ml}$ of ethanol, 0.05 mole formaldehyde and 0.05 mole of respective primary and/ secondary amine were added. The mixture was stirr for $1 \mathrm{~h}$ and then allowed to reflux for 7-8 $\mathrm{h}$ at $75{ }^{\circ} \mathrm{C}$. A clear solution was obtained. The completion of reaction was monitored by TLC. The reaction mixture was kept at $5{ }^{\circ} \mathrm{C}$ for overnight; the precipitates formed were filtered, washed with acetone and dried.

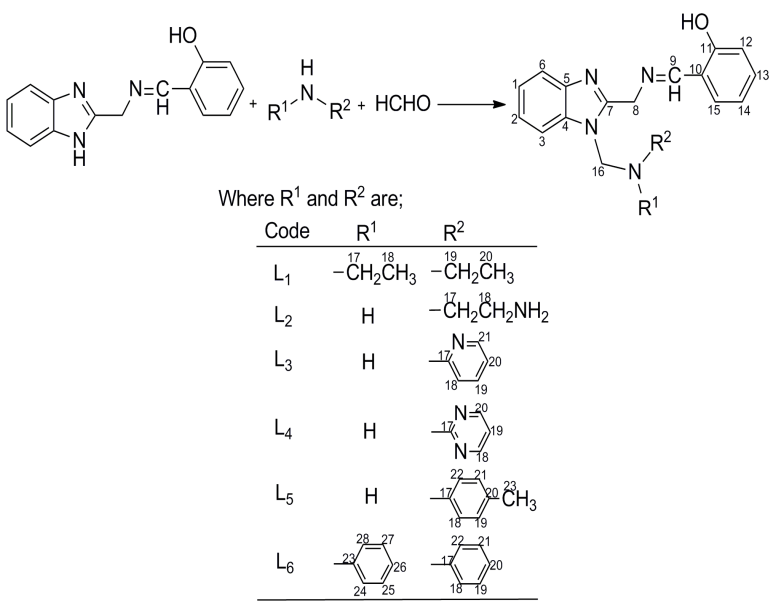

Scheme 2. Synthesis of Mannich bases.

\subsection{Synthesis of metal complexes (Scheme 3)}

To a hot magnetically stirred THF solution of Mannich bases $\left(\mathrm{L}_{1}-\mathrm{L}_{6}\right)(0.1$ mole $)$, a methanolic solution of metal(II) salts $(0.05$ mole $)$ was added. The mixture was then refluxed for $2 \mathrm{~h}$. A clear solution was obtained. The completion of reaction was monitored by TLC. The solution obtained was cooled at room temperature, precipitates appeared were filtered and washed with acetone and dried.

$$
\begin{aligned}
& \mathrm{MCl}_{2}+2 \text { Ligands }(\mathrm{L}) \longrightarrow\left[\mathrm{M}(\mathrm{L})_{2}\left(\mathrm{OH}_{2}\right)_{2}\right] \\
& \text { Where; } \\
& \begin{array}{l}
\mathrm{M}=\mathrm{Co}(\mathrm{II}), \mathrm{Ni}(\mathrm{II}), \mathrm{Cu}(\mathrm{II}), \mathrm{Zn}(\mathrm{II}) \\
\mathrm{L}=\mathrm{L}_{1}, \mathrm{~L}_{2}, \mathrm{~L}_{3}, \mathrm{~L}_{4}, \mathrm{~L}_{5}, \mathrm{~L}_{6} \\
\text { Scheme 3. Synthesis of Metal complexes. }
\end{array}
\end{aligned}
$$

\section{5. (E)-2-((((1H-Benzo[d] Imidazol-2-yl) Methyl)Imino) Methyl) Phenol}

Yellow solid; yield 70\%; m.p: $208-210{ }^{\circ} \mathrm{C}$; IR (KBr): 3474 (-NH Stretching), $3438(-\mathrm{OH}), 3055$ (Aromatic $\left.-\mathrm{CH}_{2}\right)$, $1648(-\mathrm{C}=\mathrm{N}), 1544$ (-NH Bending), $1346(\mathrm{C}-\mathrm{N}), 1236$ (C-O, phenolic); ${ }^{1} \mathrm{H}-\mathrm{NMR}$ (DMSO-d $\left.\mathrm{D}_{6}\right): \delta 11.92(\mathrm{~b}, 1 \mathrm{H}, \mathrm{OH}), 8.81$ $(\mathrm{s}, 1 \mathrm{H},-\mathrm{CH}=\mathrm{N}), 7.42(\mathrm{~m}, 4 \mathrm{H}), 7.66(\mathrm{~d}, J=7.4 \mathrm{~Hz}, 1 \mathrm{H}$, aromatic), 7.01 (d, $J=4.5 \mathrm{~Hz}, 3 \mathrm{H}), 9.76(\mathrm{~b}, 1 \mathrm{H},-\mathrm{NH}), 5.09$ (s, 2H, $\left.-\mathrm{CH}_{2}-\right) ;{ }^{13} \mathrm{C}$ NMR (DMSO-d $): \delta 162.1,131.6,124.2$, 119.3, 116.5, (C18, C14, C13, C15, C17, Phenol ring), $158.3(\mathrm{C} 12$, imine, $-\mathrm{CH}=\mathrm{N}), 140.5(\mathrm{C} 8$, imidazole, $\mathrm{N}=\mathrm{C}-\mathrm{N})$, 121.6, 109.8, 136.2 (C1, C3, C5, benzimidazole, Phenyl ring), 54.7 (C10, Aliphatic $\mathrm{CH}_{2}$ ); Mass spectrum (ESI) $[\mathrm{M}]^{+}=251$; Anal. Calcd. for $\mathrm{C}_{15} \mathrm{H}_{13} \mathrm{~N}_{3} \mathrm{O}$ (251.28) (\%): C, 71.69; H, 5.21; N, 16.72. Found (\%): C, 71.60; H, 5.30; N, 16.80 .

\section{6. (E)-2-((((1-((Diethylamino) Methyl)-1H-Benzo [D]imidazol-2-yl) Methyl)Imino)Methyl) Phenol $\left(L_{1}\right)$}

Dark brown solid; yield $72 \%$; d.p: $220-224{ }^{\circ} \mathrm{C}$; IR (KBr): $3442(\mathrm{OH}), 3055$ (Aromatic $\left.\mathrm{CH}_{2}\right), 2954\left(\mathrm{CH}_{3}\right.$ Stretching), $2847\left(\mathrm{CH}_{2}\right.$ Stretching), $1655(\mathrm{C}=\mathrm{N}), 1355$ (C-N, Amine), 1242 (C-O, phenolic); ${ }^{1} \mathrm{H}-\mathrm{NMR}$ (DMSO-d ${ }_{6}$ ): $\delta 12.86$ (b, 
$1 \mathrm{H}, \mathrm{OH}), 8.82(\mathrm{~s}, 1 \mathrm{H},-\mathrm{CH}=\mathrm{N}), 7.47(\mathrm{~m}, 4 \mathrm{H}), 7.66(\mathrm{~d}, J=$ $7.4 \mathrm{~Hz}, 1 \mathrm{H}$, aromatic), $7.01(\mathrm{~d}, J=4.5 \mathrm{~Hz}, 3 \mathrm{H}), 5.05(\mathrm{~s}, 2 \mathrm{H}$, $\left.-\mathrm{CH}_{2}{ }^{-}\right), 4.92\left(\mathrm{~s}, 2 \mathrm{H},-\mathrm{CH}_{2}^{-}\right), 2.32\left(\mathrm{~m}, 4 \mathrm{H}, \mathrm{CH}_{2}\right), 1.27(\mathrm{~m}$, $\left.6 \mathrm{H}, \mathrm{CH}_{3}\right) ;{ }^{13} \mathrm{C}$ NMR (DMSO-d 6 ): $\delta 162.1,132.6,124.2$, 117.3, 116.5, (C18, C14, C13, C15, C17, Phenol ring), $155.3(\mathrm{C} 12$, imine, $-\mathrm{CH}=\mathrm{N}), 140.5(\mathrm{C} 8$, imidazole, $\mathrm{N}=\mathrm{C}-\mathrm{N})$, 122.6, 109.8, 134.2 (C1, C3, C5, benzimidazole, Phenyl ring), $54.7\left(\mathrm{C} 10\right.$, Aliphatic $\left.\mathrm{CH}_{2},-\mathrm{N}-\mathrm{CH}_{2}-\mathrm{C}\right), 79.2$ (C20, Aliphatic $\left.\mathrm{CH}_{2},-\mathrm{N}-\mathrm{CH}_{2}-\mathrm{N}\right), 43.1\left(\mathrm{C} 22\right.$, Ethyl $\left.\mathrm{CH}_{2}\right), 17.2$ $\left(\mathrm{C} 25, \mathrm{CH}_{3}\right)$; Mass spectrum (ESI) $[\mathrm{M}]^{+}=336$; Anal. Calcd. for $\mathrm{C}_{20} \mathrm{H}_{24} \mathrm{~N}_{4} \mathrm{O}$ (336.43) (\%): C, 71.40; H, 7.19; N, 16.65. Found (\%): C, 72.42; H, 7.23; N, 16.67; ${ }^{1} \mathrm{H}-\mathrm{NMR}$ of $\mathrm{Zn}(\mathrm{II})$ complex $\left(\mathrm{DMSO}_{-}\right): \delta 9.41(\mathrm{~s}, 1 \mathrm{H},-\mathrm{CH}=\mathrm{N}), 7.71(\mathrm{~m}, 4 \mathrm{H})$, $8.02(\mathrm{~d}, J=7.4 \mathrm{~Hz}, 1 \mathrm{H}$, aromatic), $7.45(\mathrm{~d}, J=4.5 \mathrm{~Hz}, 3 \mathrm{H})$, $5.58\left(\mathrm{~s}, 2 \mathrm{H},-\mathrm{CH}_{2}-\right), 5.41\left(\mathrm{~s}, 2 \mathrm{H},-\mathrm{CH}_{2}-\right), 2.77\left(\mathrm{~m}, 4 \mathrm{H}, \mathrm{CH}_{2}\right)$, $1.57\left(\mathrm{~m}, 6 \mathrm{H}, \mathrm{CH}_{3}\right) ;{ }^{13} \mathrm{C}$ NMR of $\mathrm{Zn}(\mathrm{II})$ complex (DMSO-d $\left.{ }_{6}\right): \delta 162.9,133.1,124.8,117.7,117.4,(\mathrm{C} 18, \mathrm{C} 14$, $\mathrm{C} 13, \mathrm{C} 15, \mathrm{C} 17$, Phenol ring), $155.7(\mathrm{C} 12$, imine, $-\mathrm{CH}=\mathrm{N})$, 141.3 (C8, imidazole, $\mathrm{N}=\mathrm{C}-\mathrm{N}), 123.3,110.2,134.7$ (C1, C3, $\mathrm{C} 5$, benzimidazole, Phenyl ring), $55.3\left(\mathrm{C} 10\right.$, Aliphatic $\mathrm{CH}_{2}$, -N-CH$-\mathrm{C}), 79.7$ (C20, Aliphatic $\left.\mathrm{CH}_{2},-\mathrm{N}-\mathrm{CH}_{2}-\mathrm{N}\right), 43.9$ $\left(\mathrm{C} 22\right.$, Ethyl $\left.\mathrm{CH}_{2}\right), 17.8\left(\mathrm{C} 25, \mathrm{CH}_{3}\right)$.

\section{7. (E)-2-((((1-(((2-Aminoethyl) Amino) Methyl)-1H- Benzo [D/Imidazol-2-yl) Methyl) Imino) Methyl) Phenol $\left(L_{2}\right)$}

Light yellow solid; yield $68 \%$; d.p: $225-228{ }^{\circ} \mathrm{C}$; IR (KBr): $3433(\mathrm{OH}), 3382\left(\mathrm{NH}\right.$ stretching), $3342\left(\mathrm{NH}_{2}\right.$ Stretching), 3055 (Aromatic $\left.\mathrm{CH}_{2}\right), 2854\left(\mathrm{CH}_{2}\right.$ Stretching), $1660(\mathrm{C}=\mathrm{N}), 1574\left(\mathrm{NH}\right.$ bending), $1564\left(\mathrm{NH}_{2}\right.$ bending), 1348 (C-N, aromatic amine), 1254 (C-O, phenolic); ${ }^{1} \mathrm{H}-\mathrm{NMR}\left(\mathrm{DMSO}-\mathrm{d}_{6}\right): \delta 12.92(\mathrm{~b}, 1 \mathrm{H}, \mathrm{OH}), 8.81(\mathrm{~s}, 1 \mathrm{H}$, $-\mathrm{CH}=\mathrm{N}), 7.38(\mathrm{~m}, 4 \mathrm{H}), 7.62(\mathrm{~d}, J=7.4 \mathrm{~Hz}, 1 \mathrm{H}$, aromatic), $6.98(\mathrm{~d}, J=4.5 \mathrm{~Hz}, 3 \mathrm{H}), 5.19\left(\mathrm{~m}, 2 \mathrm{H}, \mathrm{NH}_{2}\right), 5.02(\mathrm{~s}, 2 \mathrm{H}$, $\left.-\mathrm{CH}_{2}-\right), 4.92\left(\mathrm{~d}, J=6.2 \mathrm{~Hz}, 2 \mathrm{H},-\mathrm{CH}_{2}-\right), 3.81(\mathrm{~b}, 1 \mathrm{H}, \mathrm{NH})$, $2.79\left(\mathrm{~m}, 2 \mathrm{H}, \mathrm{CH}_{2}\right), 2.57\left(\mathrm{~m}, 2 \mathrm{H},-\mathrm{CH}_{2}-\right) ;{ }^{13} \mathrm{C} \mathrm{NMR}$ (DMSO-d $\left.{ }_{6}\right): \delta 164.4,131.5,124.4,119.2,116.5,(\mathrm{C} 18, \mathrm{C} 14$, C13, C15, C17, Phenol ring), $157.5(\mathrm{C} 12$, imine, $-\mathrm{CH}=\mathrm{N})$, 144.2 (C8, imidazole, $\mathrm{N}=\mathrm{C}-\mathrm{N}), 122.6,109.8,136.2(\mathrm{C} 1, \mathrm{C} 3$, $\mathrm{C} 5$, benzimidazole, Phenyl ring), 64.1 (C20, Aliphatic $\mathrm{CH}_{2}$, $\left.-\mathrm{N}-\mathrm{CH}_{2}-\mathrm{N}\right), \quad 53.4$ (C10, Aliphatic $\left.\mathrm{CH}_{2}\right), 42.1$ (C22, Aliphatic $\left.\mathrm{CH}_{2},-\mathrm{N}-\mathrm{CH}_{2}-\mathrm{C}\right), 37.3\left(\mathrm{C} 24\right.$, Aliphatic $\mathrm{CH}_{2}$, ${ }_{2} \mathrm{HN}-\mathrm{CH}_{2}-\mathrm{C}$ ); Mass spectrum (ESI) $[\mathrm{M}]^{+}=323$; Anal. Calcd. for $\mathrm{C}_{18} \mathrm{H}_{21} \mathrm{~N}_{5} \mathrm{O}$ (323.39) (\%): C, 66.85; H, 6.54; N, 21.65. Found (\%): C, 66.87; H, 6.57; N, 21.66; ${ }^{1} \mathrm{H}-\mathrm{NMR}$ of $\mathrm{Zn}(\mathrm{II})$ complex $\left(\mathrm{DMSO}-\mathrm{d}_{6}\right): \delta 9.38(\mathrm{~s}, 1 \mathrm{H},-\mathrm{CH}=\mathrm{N}), 7.79$ (m, 4H), 8.02 (d, $J=7.4 \mathrm{~Hz}, 1 \mathrm{H}$, aromatic), 7.48 (d, $J=4.5$ $\mathrm{Hz}, 3 \mathrm{H}), 5.49\left(\mathrm{~m}, 2 \mathrm{H}, \mathrm{NH}_{2}\right), 5.33\left(\mathrm{~s}, 2 \mathrm{H},-\mathrm{CH}_{2}-\right), 5.21(\mathrm{~d}, J$ $\left.=6.2 \mathrm{~Hz}, 2 \mathrm{H},-\mathrm{CH}_{2-}\right), 4.03(\mathrm{~b}, 1 \mathrm{H}, \mathrm{NH}), 3.04\left(\mathrm{~m}, 2 \mathrm{H}, \mathrm{CH}_{2}\right)$, $2.98\left(\mathrm{~m}, 2 \mathrm{H},-\mathrm{CH}_{2}-\right) ;{ }^{13} \mathrm{C}$ NMR of $\mathrm{Zn}(\mathrm{II})$ complex (DMSO-d $\left.{ }_{6}\right): \delta 164.9,132.3,124.8,119.6,116.9,(\mathrm{C} 18, \mathrm{C} 14$, $\mathrm{C} 13, \mathrm{C} 15, \mathrm{C} 17$, Phenol ring), $158.2(\mathrm{C} 12$, imine, $-\mathrm{CH}=\mathrm{N})$, 145.1 (C8, imidazole, $\mathrm{N}=\mathrm{C}-\mathrm{N}), 123.3,110.3,136.9(\mathrm{C} 1, \mathrm{C} 3$, $\mathrm{C} 5$, benzimidazole, Phenyl ring), 64.8 (C20, Aliphatic $\mathrm{CH}_{2}$, $\left.-\mathrm{N}-\mathrm{CH}_{2}-\mathrm{N}\right), \quad 53.6 \quad\left(\mathrm{C} 10\right.$, Aliphatic $\left.\mathrm{CH}_{2}\right), 42.7$ (C22, Aliphatic $\left.\mathrm{CH}_{2},-\mathrm{N}-\mathrm{CH}_{2}-\mathrm{C}\right), 37.8\left(\mathrm{C} 24\right.$, Aliphatic $\mathrm{CH}_{2}$, ${ }_{2} \mathrm{HN}-\mathrm{CH}_{2}-\mathrm{C}$ ).

\section{8. (E)-2-((((1-(( Pyridin-2- Ylamino) Methyl)-1H-Benzo [d] Imidazol-2-yl) Methyl) Imino) Methyl) Phenol $\left(L_{3}\right)$}

Cream color solid; yield $70 \%$; d.p: $237-240{ }^{\circ} \mathrm{C}$; IR $(\mathrm{KBr})$ : $3432(\mathrm{OH}), 3396\left(\mathrm{NH}\right.$ stretching), 3055 (Aromatic $\mathrm{CH}_{2}$ ), $2867\left(\mathrm{CH}_{2}\right.$ stretching), $1653(\mathrm{C}=\mathrm{N}), 1590$ ( $\mathrm{NH}$ bending), 1348s (C-N), 1248 (C-O, phenolic); ${ }^{1} \mathrm{H}-\mathrm{NMR}$ (DMSO-d $\mathrm{d}_{6}$ ): $\delta 12.85(\mathrm{~b}, 1 \mathrm{H}, \mathrm{OH}), 8.79(\mathrm{~s}, 1 \mathrm{H},-\mathrm{CH}=\mathrm{N}), 8.02(\mathrm{~d}, 1 \mathrm{H}$, aromatic), $7.52(\mathrm{~m}, 3 \mathrm{H}), 7.42(\mathrm{~m}, 4 \mathrm{H}), 7.66(\mathrm{~d}, J=6.4 \mathrm{~Hz}$, $1 \mathrm{H}$, aromatic), $6.97(\mathrm{~d}, J=4.5 \mathrm{~Hz}, 3 \mathrm{H}), 5.09\left(\mathrm{~s}, 2 \mathrm{H},-\mathrm{CH}_{2}-\right)$, $4.87\left(\mathrm{~d}, J=6.4 \mathrm{~Hz}, 2 \mathrm{H},-\mathrm{CH}_{2}\right.$ ), $3.58(\mathrm{~b}, 1 \mathrm{H}, \mathrm{NH}) ;{ }^{13} \mathrm{C}$ NMR $\left(\right.$ DMSO-d $\left._{6}\right): \delta 161.2,131.6,124.2,119.2,116.1$, (C18, C14, C13, C15, C17, Phenol ring), 156.1 (C12, imine, $-\mathrm{CH}=\mathrm{N}), 141.2(\mathrm{C} 8$, imidazole, $\mathrm{N}=\mathrm{C}-\mathrm{N}),(152.3,145.7$, 138.5, 123.6, 121.3 (C22, C27, C25, C26, C24, 2-Pyridine ring), 121.6, 109.8, 134.2 (C1, C3, C5, benzimidazole, Phenyl ring), 74.2 (C20, Aliphatic $\left.\mathrm{CH}_{2},-\mathrm{N}-\mathrm{CH}_{2}-\mathrm{N}\right), 54.7$ (C10, Aliphatic $\mathrm{CH}_{2}$ ); Mass spectrum (ESI) $[\mathrm{M}]^{+}=357$; Anal. Calcd. for $\mathrm{C}_{21} \mathrm{H}_{19} \mathrm{~N}_{5} \mathrm{O}$ (357.40) (\%): C, 70.57; H, 5.35; $\mathrm{N}, 19.59$. Found (\%): C, 70.62; H, 5.41; N, 19.62;); ${ }^{1} \mathrm{H}-\mathrm{NMR}$ of $\mathrm{Zn}(\mathrm{II})$ complex (DMSO-d $\left.\mathrm{d}_{6}\right): \delta 9.40(\mathrm{~s}, 1 \mathrm{H}$, $-\mathrm{CH}=\mathrm{N}), 8.32(\mathrm{~d}, 1 \mathrm{H}$, aromatic), $7.86(\mathrm{~m}, 3 \mathrm{H}), 7.74(\mathrm{~m}$, $4 \mathrm{H}), 8.09(\mathrm{~d}, J=6.4 \mathrm{~Hz}, 1 \mathrm{H}$, aromatic), $7.37(\mathrm{~d}, J=4.5 \mathrm{~Hz}$, $3 \mathrm{H}), 5.39$ (s, 2H, $\left.-\mathrm{CH}_{2^{-}}\right), 5.19\left(\mathrm{~d}, \mathrm{~J}=6.4 \mathrm{~Hz}, 2 \mathrm{H},-\mathrm{CH}_{2^{-}}\right)$, 3.91 (b, 1H, NH); ${ }^{13} \mathrm{C}$ NMR of $\mathrm{Zn}(\mathrm{II})$ complex (DMSO-d $\mathrm{d}_{6}$ ): $\delta$ 161.8, 132.1, 124.8, 119.7, 116.4, (C18, C14, C13, C15, $\mathrm{C} 17$, Phenol ring), $156.6(\mathrm{C} 12$, imine, $-\mathrm{CH}=\mathrm{N}), 141.8(\mathrm{C} 8$, imidazole, $\mathrm{N}=\mathrm{C}-\mathrm{N}), 152.8,146.2,138.8,123.9,121.7$ (C22, C27, C25, C26, C24, 2-Pyridine ring), 122.1, 110.3, 134.4 (C1, C3, C5, benzimidazole, Phenyl ring), 74.7 (C20, Aliphatic $\left.\mathrm{CH}_{2},-\mathrm{N}-\mathrm{CH}_{2}-\mathrm{N}\right), 55.2\left(\mathrm{C} 10\right.$, Aliphatic $\left.\mathrm{CH}_{2}\right)$.

\section{9. (E)-2-((((1-((Pyrimidin-2-Ylamino) Methyl)-1H- Benzo (dlImidazol-2-yl) Methyl) Imino) Methyl) Phenol $\left(L_{4}\right)$}

White solid; yield $65 \%$; d.p: $238-242{ }^{\circ} \mathrm{C}$; IR (KBr): 3446 $(\mathrm{OH}), 3396$ (NH stretching), 3055 (Aromatic $\mathrm{CH}_{2}$ ), 2862 $\left(\mathrm{CH}_{2}\right.$ stretching), $1651(\mathrm{C}=\mathrm{N}), 1590(\mathrm{NH}$ bending), 1350 (C-N), 1244 (C-O, phenolic); ${ }^{1} \mathrm{H}-\mathrm{NMR}$ (DMSO-d ${ }_{6}$ ): $\delta$ $12.81(\mathrm{~b}, 1 \mathrm{H}, \mathrm{OH}), 8.95(\mathrm{~s}, 1 \mathrm{H},-\mathrm{CH}=\mathrm{N}), 8.32(\mathrm{~d}, J=7.2$ $\mathrm{Hz}, 2 \mathrm{H}), 7.66(\mathrm{~d}, J=6.5 \mathrm{~Hz}, 1 \mathrm{H}$, aromatic), $7.42(\mathrm{~m}, 4 \mathrm{H})$, $7.14(\mathrm{~m}, 1 \mathrm{H}$, aromatic), $6.97(\mathrm{~d}, J=4.5 \mathrm{~Hz}, 3 \mathrm{H}), 5.09(\mathrm{~s}$, $\left.2 \mathrm{H},-\mathrm{CH}_{2}-\right), 4.82\left(\mathrm{~d}, J=6.2 \mathrm{~Hz}, 2 \mathrm{H},-\mathrm{CH}_{2}-\right), 4.15(\mathrm{~b}, 1 \mathrm{H}$, $\mathrm{NH}) ;{ }^{13} \mathrm{C}$ NMR (DMSO- $\left.\mathrm{d}_{6}\right): \delta 173.5,154.2,112.2$, (C22, C27, C26, Pyrimidine ring), 162.1, 131.6, 120.3, 119.1, 116.5, (C18, C14, C13, C15, C17, Phenol ring), 158.3 (C12, imine, $-\mathrm{CH}=\mathrm{N}), 140.5(\mathrm{C} 8$, imidazole, $\mathrm{N}=\mathrm{C}-\mathrm{N}), 122.6$, 109.8, 136.2 (C1, C3, C5, benzimidazole, Phenyl ring), $63.5\left(\mathrm{C} 20\right.$, Aliphatic $\left.\mathrm{CH}_{2},-\mathrm{N}-\mathrm{CH}_{2}-\mathrm{N}\right), 54.7$ (C10, Aliphatic $\mathrm{CH}_{2}$ ); Mass spectrum (ESI) $[\mathrm{M}]^{+}=358$; Anal. Calcd. for $\mathrm{C}_{20} \mathrm{H}_{18} \mathrm{~N}_{6} \mathrm{O}$ (358.39) (\%): C, 67.02; H, 5.06; N, 23.49. Found (\%): C, 67.19; H, 5.09; N, 23.53; ${ }^{1} \mathrm{H}-\mathrm{NMR}$ of $\mathrm{Zn}(\mathrm{II})$ complex $\left(\right.$ DMSO-d $\left._{6}\right): \delta 9.55(\mathrm{~s}, 1 \mathrm{H},-\mathrm{CH}=\mathrm{N}), 8.64(\mathrm{~d}, J=$ $7.2 \mathrm{~Hz}, 2 \mathrm{H}), 7.98$ (d, $J=6.5 \mathrm{~Hz}, 1 \mathrm{H}$, aromatic), $7.74(\mathrm{~m}$, $4 \mathrm{H}), 7.45(\mathrm{~m}, 1 \mathrm{H}$, aromatic), $7.42(\mathrm{~d}, J=4.5 \mathrm{~Hz}, 3 \mathrm{H})$, $5.39\left(\mathrm{~s}, 2 \mathrm{H},-\mathrm{CH}_{2}-\right), 5.17\left(\mathrm{~d}, J=6.2 \mathrm{~Hz}, 2 \mathrm{H},-\mathrm{CH}_{2}-\right), 4.55(\mathrm{~b}$, 
$1 \mathrm{H}, \mathrm{NH}) ;{ }^{13} \mathrm{C}$ NMR of $\mathrm{Zn}(\mathrm{II})$ complex (DMSO-d 6 ): $\delta 173.9$, 154.5, 112.6, (C22, C27, C26, Pyrimidine ring), 162.9, 132.2, 120.7, 119.9, 117.2, (C18, C14, C13, C15, C17, Phenol ring), 158.7 (C12, imine, $-\mathrm{CH}=\mathrm{N}), 141.3(\mathrm{C} 8$, imidazole, $\mathrm{N}=\mathrm{C}-\mathrm{N}), 123.1,110.2,136.7$ (C1, C3, C5, benzimidazole, Phenyl ring), 64.6 (C20, Aliphatic $\mathrm{CH}_{2}$, -N-CH $2-\mathrm{N}), 55.2\left(\mathrm{C} 10\right.$, Aliphatic $\left.\mathrm{CH}_{2}\right)$.

\subsection{0. (E)-2-((((1-((p-Tolylamino) Methyl)-1H-Benzo[d] Imidazol-2-yl) Methyl) Imino) Methyl) Phenol $\left(L_{5}\right)$}

Off-white solid; yield: $69 \%$; d.p: $240-242{ }^{\circ} \mathrm{C}$; IR (KBr): $3443(\mathrm{OH}), 3392\left(\mathrm{NH}\right.$ stretching), 3046 (Aromatic $\mathrm{CH}_{2}$ ), $2940\left(\mathrm{CH}_{3}\right.$ stretching $), 2862\left(\mathrm{CH}_{2}\right.$ stretching $), 1656(\mathrm{C}=\mathrm{N})$, 1586 (NH bending), 1352 (C-N), 1246 (C-O, phenolic); ${ }^{1} \mathrm{H}-\mathrm{NMR}\left(\mathrm{DMSO}_{\mathrm{d}}\right.$ ): $\delta 12.87(\mathrm{~b}, 1 \mathrm{H}, \mathrm{OH}), 8.81(\mathrm{~s}, 1 \mathrm{H}$, $-\mathrm{CH}=\mathrm{N}), 7.74(\mathrm{~d}, J=5.4 \mathrm{~Hz}, 1 \mathrm{H}$, aromatic), $7.51(\mathrm{~m}, 4 \mathrm{H})$, $7.12(\mathrm{~d}, J=8.2 \mathrm{~Hz}, 2 \mathrm{H}), 6.87(\mathrm{~d}, J=4.5 \mathrm{~Hz}, 3 \mathrm{H}), 6.32(\mathrm{~d}$, $2 \mathrm{H}), 5.09\left(\mathrm{~s}, 2 \mathrm{H},-\mathrm{CH}_{2}^{-}\right), 4.73\left(\mathrm{~d}, J=6.5 \mathrm{~Hz}, 2 \mathrm{H},-\mathrm{CH}_{2}-\right)$, 3.27 (b, 1H, NH), $2.45\left(\mathrm{~s}, 3 \mathrm{H}, \mathrm{CH}_{3}\right) ;{ }^{13} \mathrm{C}$ NMR (DMSO-d 6 ): $\delta$ 162.1, 131.6, 122.3, 119.3, 116.5, (C18, C14, C13, C15, $\mathrm{C} 17$, Phenol ring), $158.3(\mathrm{C} 12$, imine, $-\mathrm{CH}=\mathrm{N}), 147.2$, 129.2, 127.2, 112.8 (C22, C25, C26, C24, Toluidine ring), 140.5 (C8, imidazole, $\mathrm{N}=\mathrm{C}-\mathrm{N}), 121.6,109.8,136.2$ (C1, C3, $\mathrm{C} 5$, benzimidazole, Phenyl ring), 68.2 (C20, Aliphatic $\mathrm{CH}_{2}$, -N-CH$-\mathrm{N}), 54.7$ (C10, Aliphatic $\left.\mathrm{CH}_{2}\right), 27.3\left(\mathrm{C} 29, \mathrm{CH}_{3}\right)$; Mass spectrum (ESI) $[\mathrm{M}]^{+}=371$; Anal. Calcd. for $\mathrm{C}_{23} \mathrm{H}_{22} \mathrm{~N}_{4} \mathrm{O}$ (370.44) (\%): C, 74.57; H, 5.98; N, 15.12 . Found (\%): C, 74.61; H, 6.05; N, 15.17; ${ }^{1} \mathrm{H}-\mathrm{NMR}$ of $\mathrm{Zn}(\mathrm{II})$ complex $($ DMSO-d 6$): \delta 9.41(\mathrm{~s}, 1 \mathrm{H},-\mathrm{CH}=\mathrm{N}), 8.09(\mathrm{~d}, J=$ $5.4 \mathrm{~Hz}, 1 \mathrm{H}$, aromatic), $7.83(\mathrm{~m}, 4 \mathrm{H}), 7.54(\mathrm{~d}, J=8.2 \mathrm{~Hz}$, 2H), $7.21(\mathrm{~d}, J=4.5 \mathrm{~Hz}, 3 \mathrm{H}), 6.69$ (d, 2H), $5.44(\mathrm{~s}, 2 \mathrm{H}$, $\left.-\mathrm{CH}_{2}-\right), 5.09$ (d, $\left.J=6.5 \mathrm{~Hz}, 2 \mathrm{H},-\mathrm{CH}_{2}-\right), 3.64(\mathrm{~b}, 1 \mathrm{H}, \mathrm{NH})$, $2.68\left(\mathrm{~s}, 3 \mathrm{H}, \mathrm{CH}_{3}\right) ;{ }^{13} \mathrm{C}$ NMR of $\mathrm{Zn}(\mathrm{II})$ complex (DMSO-d $\mathrm{d}_{6}$ ): $\delta$ 162.7, 132.4, 122.7, 119.8, 117.2, (C18, C14, C13, C15, $\mathrm{C} 17$, Phenol ring), $158.9(\mathrm{C} 12$, imine, $-\mathrm{CH}=\mathrm{N}), 147.7$, 129.8, 128.1, 113.4 (C22, C25, C26, C24, Toluidine ring), 141.2 (C8, imidazole, $\mathrm{N}=\mathrm{C}-\mathrm{N}), 122.4,111.2,136.9$ (C1, C3, C5, benzimidazole, Phenyl ring), 69.5 (C20, Aliphatic $\mathrm{CH}_{2}$, -N-CH $2-\mathrm{N}), 55.6\left(\mathrm{C} 10\right.$, Aliphatic $\left.\mathrm{CH}_{2}\right), 27.8\left(\mathrm{C} 29, \mathrm{CH}_{3}\right)$.

\subsection{1. (E)-2-((((1-((Diphenylamino) Methyl)-1H-Benzo[d] Imidazol-2-yl) Methyl) Imino) Methyl) Phenol (Lo}

Light skin solid; yield: $64 \%$; d.p: $260-262{ }^{\circ} \mathrm{C}$; IR (KBr): $3440(\mathrm{OH}), 3053$ (Aromatic $\left.\mathrm{CH}_{2}\right), 2854\left(\mathrm{CH}_{2}\right.$ stretching), $1651(\mathrm{C}=\mathrm{N}), 1544$ (NH bending), $1309(\mathrm{C}-\mathrm{N}), 1236(\mathrm{C}-\mathrm{O}$, phenolic); ${ }^{1} \mathrm{H}-\mathrm{NMR}$ (DMSO-d $\left.{ }_{6}\right): \delta 12.90(\mathrm{~b}, 1 \mathrm{H}, \mathrm{OH}), 8.74$ $(\mathrm{s}, 1 \mathrm{H},-\mathrm{CH}=\mathrm{N}), 7.59(\mathrm{~d}, J=6.5 \mathrm{~Hz}, 1 \mathrm{H}$, aromatic $), 7.42(\mathrm{~m}$, $4 \mathrm{H}), 7.01(\mathrm{~d}, J=4.5 \mathrm{~Hz}, 3 \mathrm{H}), 6.67(\mathrm{~m}, 10 \mathrm{H}$, phenyl ring), 5.09 (s, 2H, - $\left.\mathrm{CH}_{2}-\right), 4.87$ (s, 2H, $\left.-\mathrm{CH}_{2}-\right), 3.17$ (b, 1H, NH); ${ }^{13} \mathrm{C}$ NMR (DMSO-d ${ }_{6}$ ): $\delta 162.1,131.6,124.2,119.3,116.5$, (C18, C14, C13, C15, C17, Phenol ring), 158.3 (C12, imine, $-\mathrm{CH}=\mathrm{N}), 143.2,127.3,120.1,114.1$ (C22, C25, C26, C24, Phenyl ring), 140.5 (C8, imidazole, $\mathrm{N}=\mathrm{C}-\mathrm{N}), 121.6,109.8$, 136.2 (C1, C3, C5, benzimidazole, Phenyl ring), 71.3 (C20, Aliphatic $\left.\mathrm{CH}_{2},-\mathrm{N}-\mathrm{CH}_{2}-\mathrm{N}\right), 54.7\left(\mathrm{C} 10\right.$, Aliphatic $\left.\mathrm{CH}_{2}\right)$; Mass spectrum (ESI) $[\mathrm{M}]^{+}=433$; Anal. Calcd. for
$\mathrm{C}_{28} \mathrm{H}_{24} \mathrm{~N}_{4} \mathrm{O}$ (432.51)(\%): C, 77.75; H, 5.59; N, 12.95 . Found (\%): C, 77.82; H, 5.60; N, 12.97; ${ }^{1} \mathrm{H}-\mathrm{NMR}$ of $\mathrm{Zn}(\mathrm{II})$ complex $\left.(\text { DMSO-d })_{6}\right): \delta 9.34(\mathrm{~s}, 1 \mathrm{H},-\mathrm{CH}=\mathrm{N}), 7.95(\mathrm{~d}, J=$ $6.5 \mathrm{~Hz}, 1 \mathrm{H}$, aromatic), $7.82(\mathrm{~m}, 4 \mathrm{H}), 7.41(\mathrm{~d}, J=4.5 \mathrm{~Hz}$, $3 \mathrm{H}), 6.99\left(\mathrm{~m}, 10 \mathrm{H}\right.$, phenyl ring), $5.41\left(\mathrm{~s}, 2 \mathrm{H},-\mathrm{CH}_{2}-\right), 5.17$ $\left(\mathrm{s}, 2 \mathrm{H},-\mathrm{CH}_{2}-\right), 3.54(\mathrm{~b}, 1 \mathrm{H}, \mathrm{NH}) ;{ }^{13} \mathrm{C} \mathrm{NMR}$ of $\mathrm{Zn}(\mathrm{II})$ complex (DMSO-d ${ }_{6}$ ): $\delta$ 162.6, 131.8, 124.6, 119.8, 117.1, (C18, C14, C13, C15, C17, Phenol ring), 159.2 (C12, imine, $-\mathrm{CH}=\mathrm{N}), 143.8,127.9,120.7,114.5$ (C22, C25, C26, C24, Phenyl ring), 141.2 (C8, imidazole, $\mathrm{N}=\mathrm{C}-\mathrm{N}), 122.3,110.1$, 136.8 (C1, C3, C5, benzimidazole, Phenyl ring), 71.7 (C20, Aliphatic $\left.\mathrm{CH}_{2},-\mathrm{N}-\mathrm{CH}_{2}-\mathrm{N}\right), 55.3\left(\mathrm{C} 10\right.$, Aliphatic $\left.\mathrm{CH}_{2}\right)$.

\subsection{Antibacterial Activity}

The in-vitro antibacterial activity of Mannich bases $\left(\mathbf{L}_{\mathbf{1}}-\mathbf{L}_{\mathbf{6}}\right)$ and their metal(II) complexes $\left(\mathbf{C}_{\mathbf{1}}-\mathbf{C}_{\mathbf{2 4}}\right)$ was assayed against two Gram-negative (Escherichia coli, Pseudomonas aeruginosa) and two Gram-positive (Staphylococcus aureus, Bacillus subtilis) bacterial strains by the reported method.[21,22] The stock solution $(1 \mathrm{mg} / \mathrm{ml})$ of the test chemical was prepared by dissolving $10 \mathrm{mg}$ of the test compound in $10 \mathrm{ml}$ of Dimethyl sulfoxide (DMSO) solvent. The stock solution was suitably diluted with sterilized distilled water to get dilution of 100,50 and $25 \mathrm{mgml}^{-1}$. Control for each dilution was prepared by diluting $10 \mathrm{ml}$ of solvent instead of stock solution with sterilized distilled water. The wells (6 $\mathrm{mm}$ in diameter) were dug in the agar media with the help of a sterile metallic borer. Two to eight hours old bacterial inocula containing approximately 104-106 colony forming units $(\mathrm{CFU} / \mathrm{mL})$ were spread on the surface of the nutrient agar with the help of a sterile cotton swab. The prepared concentrations of the test sample were introduced in the respective wells. Other wells supplemented with DMSO and reference antibacterial drug, Gentamycin, served as negative and positive controls, respectively. The plates were incubated immediately at $37^{\circ} \mathrm{C}$ for $24 \mathrm{~h}$. Activity was determined by measuring the diameter of zones showing complete inhibition ( $\mathrm{mm}$ ). In order to clarify any effect of DMSO in the biological screening, separate studies were carried out with the solutions alone of DMSO and they showed no activity against any bacterial strains.

\subsection{Antifungal Activity}

All the compounds $\left(\mathrm{L}_{1}-\mathrm{L}_{6}\right)$ and their metal(II) complexes $\left(\mathrm{C}_{1}-\mathrm{C}_{24}\right)$ were studied against five fungal cultures (Aspergillus niger, Penicillium expansum, Rhizopus nigricans, Trichoderna lignorum, Botrydepladia thiobromine) for Antifungal activities. Sabouraud dextrose agar (Oxoid, Hampshire, England) was seeded with $105 \mathrm{cfu}$ $\mathrm{ml}^{-1}$ fungal spore suspensions and transferred to Petri plates. The stock solution of test chemical was prepared and diluted to 100,50 and $25 \mathrm{mg} \mathrm{ml}^{-1}$. Discs soaked in $20 \mathrm{ml}$ of prepared concentrations of all compounds were placed at different positions on the agar surface. The plates were incubated at $32{ }^{\circ} \mathrm{C}$. The percentage inhibition was 
calculated after seven days and compared with standard drugs Fluconazole.[23]

\subsection{In Vitro Cytotoxicity}

The synthesized compounds and their Zn(II), Co(II), $\mathrm{Cu}(\mathrm{II})$ and $\mathrm{Ni}(\mathrm{II})$ complexes were screened for their cytotoxicity (brine shrimp bioassay) by using the protocol of Meyer et al.[24] Brine shrimp (Artemia salina leach) eggs were hatched in a shallow rectangular plastic dish (22 x $32 \mathrm{~cm}$ ) filled with artificial seawater, which was prepared with a commercial salt mixture and double distilled water. An unequal partition was made in the plastic dish with the help of a perforated device. Approximately $50 \mathrm{mg}$ of eggs were sprinkled into the large compartment, which was darkened while the minor compartment was open to ordinary light. After two days nauplii were collected by a pipette from the lighted side. A sample of the test compound was prepared by dissolving $20 \mathrm{mg}$ of each compound in $2 \mathrm{ml}$ of DMSO. From this stock solution 100, 50 and $25 \mathrm{mgml}^{-1}$ were transferred to nine vials (three for each dilutions were used for each test sample and LD50 is the mean of three values) and one vial was kept as control having $2 \mathrm{ml}$ of DMSO only. The solvent was allowed to evaporate overnight. After two days, when shrimp larvae were ready, $1 \mathrm{ml}$ of seawater and 10 shrimps were added to each vial (30 shrimps/dilution) and the volume was adjusted with seawater to $5 \mathrm{ml}$ per vial. After $24 \mathrm{~h}$ the number of survivors was counted. Data were analyzed by a Finney computer program to determine the $\mathrm{LD}_{50}$ values.[25]

\section{Results and Discussion}

\subsection{IR Spectra}

The important IR spectral bands of the Mannich and Schiff bases and their metal complexes along with their tentative assignments are given in the experimental and Table 1.

The ligands show a broad band at $3432-3446 \mathrm{~cm}^{-1}$ and sharp bands at $1648-1660 \mathrm{~cm}^{-1}, 1236-1254 \mathrm{~cm}^{-1}$, assigned to H-bonded $-\mathrm{OH}, \mathrm{v}(\mathrm{C}=\mathrm{N})$ and phenolic $\mathrm{v}(\mathrm{C}-\mathrm{O})$ stretching vibrations respectively. In the complexes, the azomethine frequency shows a downfall $\left(15-30 \mathrm{~cm}^{-1}\right)$ indicating coordination through $\mathrm{N}$ atom. This is further supported by the appearance of new bands at $450-486 \mathrm{~cm}^{-1}$ due to $v(\mathrm{M}-\mathrm{N})$ bond.[26]

The shifting of medium to high intensity bands of phenolic C-O bond towards higher frequency (1339-1368 $\mathrm{cm}^{-1}$ ) and appearance of new bands at $510-540 \mathrm{~cm}^{-1}$ support the formation of M-O bond via de-protonation.[27]

The presence of coordinated water molecule in the complex is indicated by the appearance of a broad band at $3226-3460 \mathrm{~cm}^{-1}$ and two weak bands in the region 754-784 $\mathrm{cm}^{-1}$ and $700-718 \mathrm{~cm}^{-1}$ due to (-OH) rocking and wagging mode of vibrations, respectively.[28]
Table 1. The important infrared frequencies (in $\mathrm{cm}^{-1}$ ) of $\mathrm{Zn}(I I)$, Co(II), $\mathrm{Cu}(\mathrm{II})$ and $\mathrm{Ni}(\mathrm{II})$ complexes.

\begin{tabular}{|c|c|c|c|c|c|}
\hline Code & Complex & $v(C=N)$ & $v(M-N)$ & $v(M-O)$ & $v(C-O)$ \\
\hline $\mathrm{C}_{1}$ & {$\left[\mathrm{Zn}\left(\mathrm{L}_{1}\right)_{2}\left(\mathrm{OH}_{2}\right)_{2}\right]$} & $1632 \mathrm{~s}$ & $455 \mathrm{~m}$ & $510 \mathrm{~m}$ & $1340 \mathrm{~m}$ \\
\hline $\mathrm{C}_{2}$ & {$\left[\mathrm{Zn}\left(\mathrm{L}_{2}\right)_{2}\left(\mathrm{OH}_{2}\right)_{2}\right]$} & $1630 \mathrm{~m}$ & $462 \mathrm{~m}$ & $540 \mathrm{~m}$ & $1349 \mathrm{~m}$ \\
\hline $\mathrm{C}_{3}$ & {$\left[\mathrm{Zn}\left(\mathrm{L}_{3}\right)_{2}\left(\mathrm{OH}_{2}\right)_{2}\right]$} & $1634 \mathrm{~m}$ & $458 \mathrm{~m}$ & $535 \mathrm{~m}$ & $1345 \mathrm{~m}$ \\
\hline $\mathrm{C}_{4}$ & {$\left[\mathrm{Zn}\left(\mathrm{L}_{4}\right)_{2}\left(\mathrm{OH}_{2}\right)_{2}\right]$} & $1628 \mathrm{~m}$ & $460 \mathrm{~m}$ & $512 \mathrm{~m}$ & $1342 \mathrm{~m}$ \\
\hline $\mathrm{C}_{5}$ & {$\left[\mathrm{Zn}\left(\mathrm{L}_{5}\right)_{2}\left(\mathrm{OH}_{2}\right)_{2}\right]$} & $1629 \mathrm{~m}$ & $454 \mathrm{~m}$ & $512 \mathrm{~m}$ & $1347 \mathrm{~m}$ \\
\hline $\mathrm{C}_{6}$ & {$\left[\mathrm{Zn}\left(\mathrm{L}_{6}\right)_{2}\left(\mathrm{OH}_{2}\right)_{2}\right]$} & $1632 \mathrm{~m}$ & $450 \mathrm{~m}$ & $516 \mathrm{~m}$ & $1351 \mathrm{~s}$ \\
\hline $\mathrm{C}_{7}$ & {$\left[\mathrm{Co}\left(\mathrm{L}_{1}\right)_{2}\left(\mathrm{OH}_{2}\right)_{2}\right]$} & $1624 \mathrm{~m}$ & $460 \mathrm{~m}$ & $520 \mathrm{~m}$ & $1348 \mathrm{~m}$ \\
\hline $\mathrm{C}_{8}$ & {$\left[\mathrm{Co}\left(\mathrm{L}_{2}\right)_{2}\left(\mathrm{OH}_{2}\right)_{2}\right]$} & $1626 \mathrm{~s}$ & $475 \mathrm{~m}$ & $514 \mathrm{~m}$ & $1346 \mathrm{~m}$ \\
\hline $\mathrm{C}_{9}$ & {$\left[\mathrm{Co}\left(\mathrm{L}_{3}\right)_{2}\left(\mathrm{OH}_{2}\right)_{2}\right]$} & $1622 \mathrm{~m}$ & $465 \mathrm{~m}$ & $524 \mathrm{~m}$ & $1353 \mathrm{~m}$ \\
\hline $\mathrm{C}_{10}$ & {$\left[\mathrm{Co}\left(\mathrm{L}_{4}\right)_{2}\left(\mathrm{OH}_{2}\right)_{2}\right]$} & $1624 \mathrm{~m}$ & $480 \mathrm{~m}$ & $522 \mathrm{~m}$ & $1351 \mathrm{~m}$ \\
\hline $\mathrm{C}_{11}$ & {$\left[\mathrm{Co}\left(\mathrm{L}_{5}\right)_{2}\left(\mathrm{OH}_{2}\right)_{2}\right]$} & $1628 \mathrm{~m}$ & $476 \mathrm{~m}$ & $518 \mathrm{~m}$ & $1339 \mathrm{~m}$ \\
\hline $\mathrm{C}_{12}$ & {$\left[\mathrm{Co}\left(\mathrm{L}_{6}\right)_{2}\left(\mathrm{OH}_{2}\right)_{2}\right]$} & $1630 \mathrm{~m}$ & $468 \mathrm{~m}$ & $516 \mathrm{~m}$ & $1346 \mathrm{~m}$ \\
\hline $\mathrm{C}_{13}$ & {$\left[\mathrm{Cu}\left(\mathrm{L}_{1}\right)_{2}\left(\mathrm{OH}_{2}\right)_{2}\right]$} & $1624 \mathrm{~s}$ & $486 \mathrm{~m}$ & $526 \mathrm{~m}$ & $1349 \mathrm{~m}$ \\
\hline $\mathrm{C}_{14}$ & {$\left[\mathrm{Cu}\left(\mathrm{L}_{2}\right)_{2}\left(\mathrm{OH}_{2}\right)_{2}\right]$} & $1620 \mathrm{~m}$ & $483 \mathrm{~m}$ & $522 \mathrm{~m}$ & $1354 \mathrm{~m}$ \\
\hline $\mathrm{C}_{15}$ & {$\left[\mathrm{Cu}\left(\mathrm{L}_{3}\right)_{2}\left(\mathrm{OH}_{2}\right)_{2}\right]$} & $1618 \mathrm{~m}$ & $486 \mathrm{~m}$ & $504 \mathrm{~m}$ & $1345 \mathrm{~m}$ \\
\hline $\mathrm{C}_{16}$ & {$\left[\mathrm{Cu}\left(\mathrm{L}_{4}\right)_{2}\left(\mathrm{OH}_{2}\right)_{2}\right]$} & $1622 \mathrm{~m}$ & $474 \mathrm{~m}$ & $540 \mathrm{~m}$ & $1349 \mathrm{~m}$ \\
\hline $\mathrm{C}_{17}$ & {$\left[\mathrm{Cu}\left(\mathrm{L}_{5}\right)_{2}\left(\mathrm{OH}_{2}\right)_{2}\right]$} & $1625 \mathrm{~m}$ & $472 \mathrm{~m}$ & $510 \mathrm{~m}$ & $1341 \mathrm{~m}$ \\
\hline $\mathrm{C}_{18}$ & {$\left[\mathrm{Cu}\left(\mathrm{L}_{6}\right)_{2}\left(\mathrm{OH}_{2}\right)_{2}\right]$} & $1618 \mathrm{~m}$ & $470 \mathrm{~m}$ & $516 \mathrm{~m}$ & $1352 \mathrm{~m}$ \\
\hline $\mathrm{C}_{19}$ & {$\left[\mathrm{Ni}\left(\mathrm{L}_{1}\right)_{2}\left(\mathrm{OH}_{2}\right)_{2}\right]$} & $1620 \mathrm{~m}$ & $482 \mathrm{~s}$ & $524 \mathrm{~m}$ & $1368 \mathrm{~m}$ \\
\hline $\mathrm{C}_{20}$ & {$\left[\mathrm{Ni}\left(\mathrm{L}_{2}\right)_{2}\left(\mathrm{OH}_{2}\right)_{2}\right]$} & $1632 \mathrm{~m}$ & $478 \mathrm{~m}$ & $529 \mathrm{~s}$ & $1356 \mathrm{~m}$ \\
\hline $\mathrm{C}_{21}$ & {$\left[\mathrm{Ni}\left(\mathrm{L}_{3}\right)_{2}\left(\mathrm{OH}_{2}\right)_{2}\right]$} & $1628 \mathrm{~s}$ & $462 \mathrm{~m}$ & $532 \mathrm{~m}$ & $1358 \mathrm{~m}$ \\
\hline $\mathrm{C}_{22}$ & {$\left[\mathrm{Ni}\left(\mathrm{L}_{4}\right)_{2}\left(\mathrm{OH}_{2}\right)_{2}\right]$} & $1632 \mathrm{~m}$ & $471 \mathrm{~m}$ & $502 \mathrm{~m}$ & $1342 \mathrm{~m}$ \\
\hline $\mathrm{C}_{23}$ & {$\left[\mathrm{Ni}\left(\mathrm{L}_{5}\right)_{2}\left(\mathrm{OH}_{2}\right)_{2}\right]$} & $1630 \mathrm{~m}$ & $485 \mathrm{~m}$ & $515 \mathrm{~m}$ & $1352 \mathrm{~m}$ \\
\hline $\mathrm{C}_{24}$ & {$\left[\mathrm{Ni}\left(\mathrm{L}_{6}\right)_{2}\left(\mathrm{OH}_{2}\right)_{2}\right]$} & $1624 \mathrm{~s}$ & $472 m$ & $506 \mathrm{~m}$ & $1346 \mathrm{~m}$ \\
\hline
\end{tabular}

\section{2. ${ }^{1}$ H NMR Spectra}

${ }^{1} \mathrm{H}$ NMR spectra of the free ligands and their diamagnetic zinc (II) complexes were recorded in DMSO- $\mathrm{d}_{6}$. The ${ }^{1} \mathrm{H}$ NMR spectral data along with the 
possible assignments is recorded in the Experimental. The ${ }^{1} \mathrm{H}$ NMR spectra of Schiff base exhibits singlet at 12.92 and $8.81 \mathrm{ppm}$ due to phenolic $\mathrm{OH}$ and $-\mathrm{CH}=\mathrm{N}$ proton respectively.[29] In addition to these signals, Mannich bases $\left(\mathrm{L}_{1}-\mathrm{L}_{6}\right)$ have shown the peak at 4.92-4.73 ppm due to methylene linkage $\left(2 \mathrm{H}, \quad-\mathrm{CH}_{2}-\right)$ formed between benzimidazole moiety and amino compound. Mannich bases reaction can be further confirmed by the absence of peak for $(-\mathrm{NH})$ secondary amino group of benzimidazole ring system.[30] A quartet peak at 2.32 and triplet peak at $1.27 \mathrm{ppm}$ indicated the presence of ethyl group in $\mathrm{L}_{1}$. In $\mathrm{L}_{2}$, multiplet at 5.19 and $2.57 \mathrm{ppm}$ indicated the presence of $-\mathrm{NH}_{2}$ and $-\mathrm{CH}_{2}$ groups respectively. In $\mathrm{L}_{3}$, presence of pyridine group indicated by the appearance of doublet peak at 8.02 and multiplet at $7.52 \mathrm{ppm}$. A 2 proton doublet at 8.32 and 1 proton multiplet at $7.14 \mathrm{ppm}$ indicated the presence of pyrimidine group in $\mathrm{L}_{4}$ Mannich base. In $\mathrm{L}_{5}$, a singlet peak at 2.45 and doublet peaks at 7.12 and $6.32 \mathrm{ppm}$ indicated the presence of $\mathrm{p}$-toluene group. A multiplet peak at $6.67 \mathrm{ppm}$ indicated the presence of phenyl group in $\mathrm{L}_{6}$. The ${ }^{1} \mathrm{H}$ NMR spectra of $\mathrm{Zn}(\mathrm{II})$ complexes lend further support to the mode of bonding discussed in their IR spectra. The coordination of the azomethine nitrogen is inferred by the downfield shift of the $-\mathrm{CH}=\mathrm{N}$ - proton signal from 8.74-8.95 ppm in the ligands to $9.34-9.55 \mathrm{ppm}$ in the complexes. The hydroxyl proton in the spectra of $\mathrm{Zn}$ (II) complexes of ligands disappeared indicating deprotonation and coordination of the oxygen with the metal ion. All other protons underwent a downfield shift by 0.3-0.6 ppm due to the increased conjugation[31] and coordination with the metal atom.

\section{3. ${ }^{13}$ C NMR Spectra}

${ }^{13} \mathrm{C}$ NMR spectra of Mannich and Schiff bases were recorded in DMSO and the data are given in the Experimental. The signal due to the azomethine carbon atom appears at $\delta 153.3-158.3 \mathrm{ppm}$ in all the prepared compounds. Additional signal in the ${ }^{13} \mathrm{C}-\mathrm{NMR}$ spectra of the Mannich bases appears at 63.5-79.2 ppm indicating the methylene linkage formed between benzimidazole ring and amino compound.[32]

Table 2. Electronic Spectral dat and magnetic moments of Mannich base metal(II) complexes

\begin{tabular}{|c|c|c|c|c|c|c|c|}
\hline Code & Mag. moments ( $\mu_{\mathrm{eff}}$ in $\mathrm{BM}$ ) & $\lambda_{\max }\left(\mathrm{cm}^{-1} \mathrm{~mol}^{-1}\right)$ & Assignment & Code & Mag. moments ( $\mu_{\text {eff }}$ in $\mathrm{BM}$ ) & $\lambda_{\max }\left(\mathrm{cm}^{-1} \mathrm{~mol}^{-1}\right)$ & Assignment \\
\hline $\mathrm{C}_{1}$ & Dia & - & - & $\mathrm{C}_{13}$ & 1.78 & 14500 & ${ }^{2} \mathrm{E}_{\mathrm{g}} \rightarrow{ }^{2} \mathrm{~T}_{2 \mathrm{~g}}$ \\
\hline $\mathrm{C}_{2}$ & Dia & - & - & $\mathrm{C}_{14}$ & 1.81 & 14800 & ${ }^{2} \mathrm{E}_{\mathrm{g}} \rightarrow{ }^{2} \mathrm{~T}_{2 \mathrm{~g}}$ \\
\hline $\mathrm{C}_{3}$ & Dia & - & - & $\mathrm{C}_{15}$ & 1.83 & 14700 & ${ }^{2} \mathrm{E}_{\mathrm{g}} \rightarrow{ }^{2} \mathrm{~T}_{2 \mathrm{~g}}$ \\
\hline $\mathrm{C}_{4}$ & Dia & - & - & $\mathrm{C}_{16}$ & 1.77 & 14800 & ${ }^{2} \mathrm{E}_{\mathrm{g}} \rightarrow{ }^{2} \mathrm{~T}_{2 \mathrm{~g}}$ \\
\hline $\mathrm{C}_{5}$ & Dia & - & - & $\mathrm{C}_{17}$ & 1.72 & 14800 & ${ }^{2} \mathrm{E}_{\mathrm{g}} \rightarrow{ }^{2} \mathrm{~T}_{2 \mathrm{~g}}$ \\
\hline \multirow[t]{2}{*}{$\mathrm{C}_{6}$} & Dia & - & - & $\mathrm{C}_{18}$ & 1.80 & 14500 & ${ }^{2} \mathrm{E}_{\mathrm{g}} \rightarrow{ }^{2} \mathrm{~T}_{2 \mathrm{~g}}$ \\
\hline & & 9300 & ${ }^{4} \mathrm{~T}_{1 \mathrm{~g}}(\mathrm{~F}) \rightarrow{ }^{4} \mathrm{~T}_{2 \mathrm{~g}}(\mathrm{~F})$ & & & 9800 & ${ }^{3} \mathrm{~A}_{2 \mathrm{~g}}(\mathrm{~F}) \rightarrow{ }^{3} \mathrm{~T}_{2 \mathrm{~g}}(\mathrm{~F})$ \\
\hline \multirow[t]{3}{*}{$\mathrm{C}_{7}$} & 4.89 & 17900 & ${ }^{4} \mathrm{~T}_{1 \mathrm{~g}}(\mathrm{~F}) \rightarrow{ }^{4} \mathrm{~A}_{2 \mathrm{~g}}(\mathrm{~F})$ & $\mathrm{C}_{19}$ & 3.10 & 15900 & ${ }^{3} \mathrm{~A}_{2 \mathrm{~g}}(\mathrm{~F}) \rightarrow{ }^{3} \mathrm{~T}_{1 \mathrm{~g}}(\mathrm{~F})$ \\
\hline & & 19200 & ${ }^{4} \mathrm{~T}_{1 \mathrm{~g}}(\mathrm{~F}) \rightarrow{ }^{4} \mathrm{~T}_{1 \mathrm{~g}}(\mathrm{P})$ & & & 24500 & ${ }^{3} \mathrm{~A}_{2 \mathrm{~g}}(\mathrm{~F}) \rightarrow{ }^{3} \mathrm{~T}_{1 \mathrm{~g}}(\mathrm{P})$ \\
\hline & & 9400 & ${ }^{4} \mathrm{~T}_{1 \mathrm{~g}}(\mathrm{~F}) \rightarrow{ }^{4} \mathrm{~T}_{2 \mathrm{~g}}(\mathrm{~F})$ & & & 9700 & ${ }^{3} \mathrm{~A}_{2 \mathrm{~g}}(\mathrm{~F}) \rightarrow{ }^{3} \mathrm{~T}_{2 \mathrm{~g}}(\mathrm{~F})$ \\
\hline \multirow[t]{3}{*}{$\mathrm{C}_{8}$} & 4.78 & 17700 & ${ }^{4} \mathrm{~T}_{1 \mathrm{~g}}(\mathrm{~F}) \rightarrow{ }^{4} \mathrm{~A}_{2 \mathrm{~g}}(\mathrm{~F})$ & $\mathrm{C}_{20}$ & 2.98 & 15400 & ${ }^{3} \mathrm{~A}_{2 \mathrm{~g}}(\mathrm{~F}) \rightarrow{ }^{3} \mathrm{~T}_{1 \mathrm{~g}}(\mathrm{~F})$ \\
\hline & & 19400 & ${ }^{4} \mathrm{~T}_{1 \mathrm{~g}}(\mathrm{~F}) \rightarrow{ }^{4} \mathrm{~T}_{1 \mathrm{~g}}(\mathrm{P})$ & & & 24600 & ${ }^{3} \mathrm{~A}_{2 \mathrm{~g}}(\mathrm{~F}) \rightarrow{ }^{3} \mathrm{~T}_{1 \mathrm{~g}}(\mathrm{P})$ \\
\hline & & 9300 & ${ }^{4} \mathrm{~T}_{1 \mathrm{~g}}(\mathrm{~F}) \rightarrow{ }^{4} \mathrm{~T}_{2 \mathrm{~g}}(\mathrm{~F})$ & & & 9400 & ${ }^{3} \mathrm{~A}_{2 \mathrm{~g}}(\mathrm{~F}) \rightarrow{ }^{3} \mathrm{~T}_{2 \mathrm{~g}}(\mathrm{~F})$ \\
\hline \multirow[t]{3}{*}{$\mathrm{C}_{9}$} & 4.82 & 17700 & ${ }^{4} \mathrm{~T}_{1 \mathrm{~g}}(\mathrm{~F}) \rightarrow{ }^{4} \mathrm{~A}_{2 \mathrm{~g}}(\mathrm{~F})$ & $\mathrm{C}_{21}$ & 3.14 & 15600 & ${ }^{3} \mathrm{~A}_{2 \mathrm{~g}}(\mathrm{~F}) \rightarrow{ }^{3} \mathrm{~T}_{1 \mathrm{~g}}(\mathrm{~F})$ \\
\hline & & 19300 & ${ }^{4} \mathrm{~T}_{1 \mathrm{~g}}(\mathrm{~F}) \rightarrow{ }^{4} \mathrm{~T}_{1 \mathrm{~g}}(\mathrm{P})$ & & & 24400 & ${ }^{3} \mathrm{~A}_{2 \mathrm{~g}}(\mathrm{~F}) \rightarrow{ }^{3} \mathrm{~T}_{1 \mathrm{~g}}(\mathrm{P})$ \\
\hline & & 9200 & ${ }^{4} \mathrm{~T}_{1 \mathrm{~g}}(\mathrm{~F}) \rightarrow{ }^{4} \mathrm{~T}_{2 \mathrm{~g}}(\mathrm{~F})$ & & & 9700 & ${ }^{3} \mathrm{~A}_{2 \mathrm{~g}}(\mathrm{~F}) \rightarrow{ }^{3} \mathrm{~T}_{2 \mathrm{~g}}(\mathrm{~F})$ \\
\hline \multirow[t]{3}{*}{$\mathrm{C}_{10}$} & 4.86 & 17900 & ${ }^{4} \mathrm{~T}_{1 \mathrm{~g}}(\mathrm{~F}) \rightarrow{ }^{4} \mathrm{~A}_{2 \mathrm{~g}}(\mathrm{~F})$ & $\mathrm{C}_{22}$ & 3.21 & 15400 & ${ }^{3} \mathrm{~A}_{2 \mathrm{~g}}(\mathrm{~F}) \rightarrow{ }^{3} \mathrm{~T}_{1 \mathrm{~g}}(\mathrm{~F})$ \\
\hline & & 19500 & ${ }^{4} \mathrm{~T}_{1 \mathrm{~g}}(\mathrm{~F}) \rightarrow{ }^{4} \mathrm{~T}_{1 \mathrm{~g}}(\mathrm{P})$ & & & 24200 & ${ }^{3} \mathrm{~A}_{2 \mathrm{~g}}(\mathrm{~F}) \rightarrow{ }^{3} \mathrm{~T}_{1 \mathrm{~g}}(\mathrm{P})$ \\
\hline & & 9300 & ${ }^{4} \mathrm{~T}_{1 \mathrm{~g}}(\mathrm{~F}) \rightarrow{ }^{4} \mathrm{~T}_{2 \mathrm{~g}}(\mathrm{~F})$ & & & 9600 & ${ }^{3} \mathrm{~A}_{2 \mathrm{~g}}(\mathrm{~F}) \rightarrow{ }^{3} \mathrm{~T}_{2 \mathrm{~g}}(\mathrm{~F})$ \\
\hline \multirow[t]{3}{*}{$\mathrm{C}_{11}$} & 4.84 & 17700 & ${ }^{4} \mathrm{~T}_{1 \mathrm{~g}}(\mathrm{~F}) \rightarrow{ }^{4} \mathrm{~A}_{2 \mathrm{~g}}(\mathrm{~F})$ & $\mathrm{C}_{23}$ & 3.27 & 15600 & ${ }^{3} \mathrm{~A}_{2 \mathrm{~g}}(\mathrm{~F}) \rightarrow{ }^{3} \mathrm{~T}_{1 \mathrm{~g}}(\mathrm{~F})$ \\
\hline & & 19500 & ${ }^{4} \mathrm{~T}_{1 \mathrm{~g}}(\mathrm{~F}) \rightarrow{ }^{4} \mathrm{~T}_{1 \mathrm{~g}}(\mathrm{P})$ & & & 24400 & ${ }^{3} \mathrm{~A}_{2 \mathrm{~g}}(\mathrm{~F}) \rightarrow{ }^{3} \mathrm{~T}_{1 \mathrm{~g}}(\mathrm{P})$ \\
\hline & & 9200 & ${ }^{4} \mathrm{~T}_{1 \mathrm{~g}}(\mathrm{~F}) \rightarrow{ }^{4} \mathrm{~T}_{2 \mathrm{~g}}(\mathrm{~F})$ & & & 9500 & ${ }^{3} \mathrm{~A}_{2 \mathrm{~g}}(\mathrm{~F}) \rightarrow{ }^{3} \mathrm{~T}_{2 \mathrm{~g}}(\mathrm{~F})$ \\
\hline \multirow[t]{2}{*}{$\mathrm{C}_{12}$} & 4.88 & 17800 & ${ }^{4} \mathrm{~T}_{1 \mathrm{~g}}(\mathrm{~F}) \rightarrow{ }^{4} \mathrm{~A}_{2 \mathrm{~g}}(\mathrm{~F})$ & $\mathrm{C}_{24}$ & 3.16 & 15700 & ${ }^{3} \mathrm{~A}_{2 \mathrm{~g}}(\mathrm{~F}) \rightarrow{ }^{3} \mathrm{~T}_{1 \mathrm{~g}}(\mathrm{~F})$ \\
\hline & & 19400 & ${ }^{4} \mathrm{~T}_{1 \mathrm{~g}}(\mathrm{~F}) \rightarrow{ }^{4} \mathrm{~T}_{1 \mathrm{~g}}(\mathrm{P})$ & & & 24500 & ${ }^{3} \mathrm{~A}_{2 \mathrm{~g}}(\mathrm{~F}) \rightarrow{ }^{3} \mathrm{~T}_{1 \mathrm{~g}}(\mathrm{P})$ \\
\hline
\end{tabular}


Misbah ur Rehman et al.: Metal-based Antimicrobial Agents: Synthesis, Characterization and Biological Studies of Mannich Base Derivatives of Benzimidazole and Their Metal Complexes

Table 3. Thermogravimetric analysis (TGA) results of Mannich base metal(II) complexes.

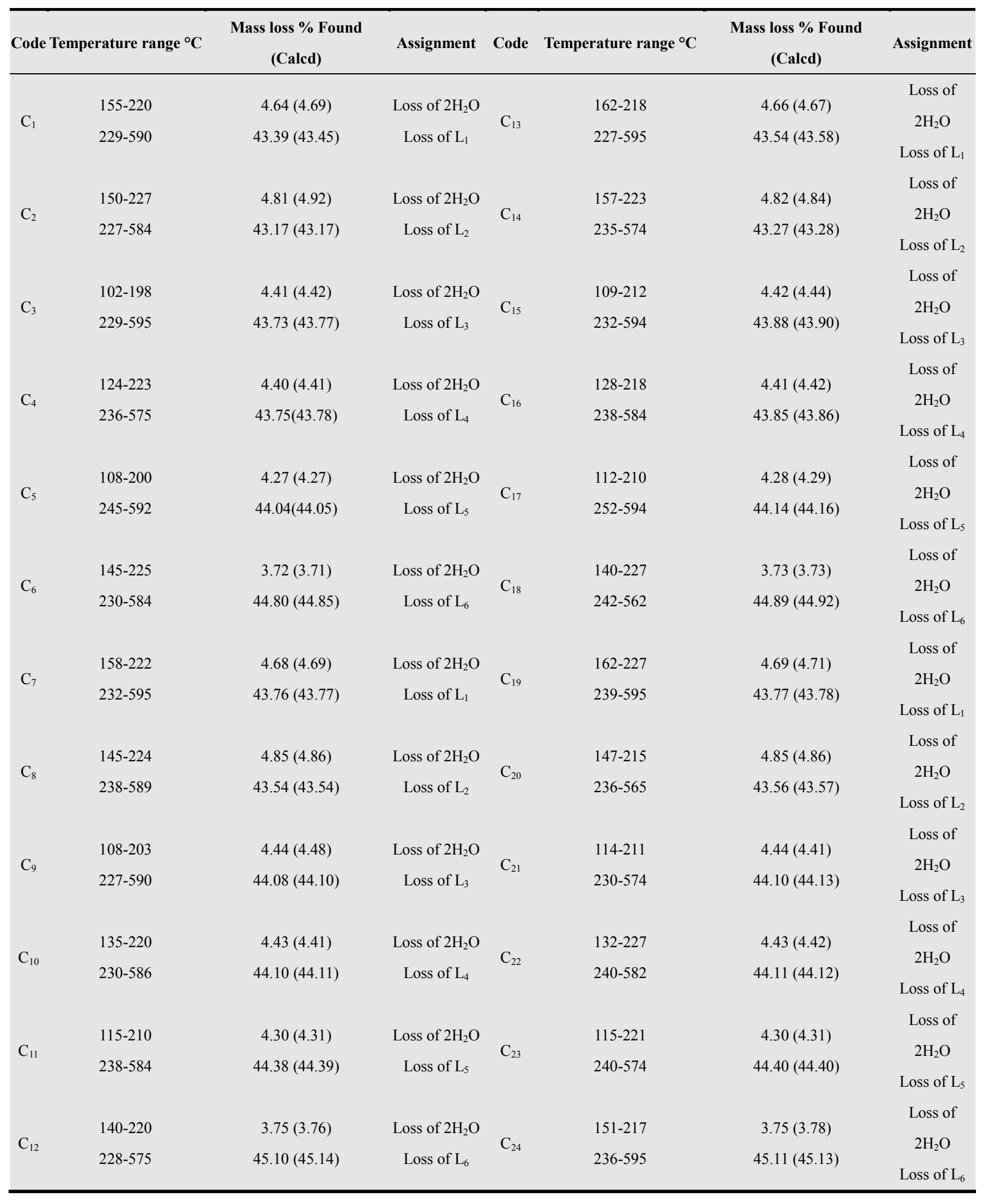

\subsection{Electronic Spectra and Magnetic Moments}

The electronic spectra if $\mathrm{Ni}(\mathrm{II})$ complexes exhibits three bands at 9400-9800, 15400-15900 and 24200-24600, which may reasonably be assignable to ${ }^{3} \mathrm{~A}_{2 \mathrm{~g}}(\mathrm{~F}) \rightarrow{ }^{3} \mathrm{~T}_{2 \mathrm{~g}}(\mathrm{~F})$,
${ }^{3} \mathrm{~A}_{2 \mathrm{~g}}(\mathrm{~F}) \rightarrow{ }^{3} \mathrm{~T}_{1 \mathrm{~g}}(\mathrm{~F}) \quad$ and $\quad{ }^{3} \mathrm{~A}_{2 \mathrm{~g}}(\mathrm{~F}) \rightarrow{ }^{3} \mathrm{~T}_{1 \mathrm{~g}}(\mathrm{P}) \quad$ transitions, respectively. The magnetic moments for $\mathrm{Ni}(\mathrm{II})$ complexes (2.98-3.27 BM) are within the range of an octahedral geometry.[33] The electronic spectra of $\mathrm{Co}(\mathrm{II})$ complexes shows absorption bands at 9200-9400, 17700-17900 and 
19200-19500 assignable to $\quad{ }^{4} \mathrm{~T}_{1 \mathrm{~g}}(\mathrm{~F}) \rightarrow{ }^{4} \mathrm{~T}_{2 \mathrm{~g}}(\mathrm{~F})$, ${ }^{4} \mathrm{~T}_{1 \mathrm{~g}}(\mathrm{~F}) \rightarrow{ }^{4} \mathrm{~A}_{2 \mathrm{~g}}(\mathrm{~F}) \quad$ and $\quad{ }^{4} \mathrm{~T}_{1 \mathrm{~g}}(\mathrm{~F}) \rightarrow{ }^{4} \mathrm{~T}_{1 \mathrm{~g}}(\mathrm{P}) \quad$ transitions, respectively. The magnetic moment values of $\mathrm{Co}(\mathrm{II})$ complexes are 4.78-4.89 BM, suggesting an octahedral geometry.[34] The observed magnetic moments for $\mathrm{Cu}$ (II) complexes are 1.72-1.83 $\mathrm{BM}$ and the band observed at 14500-14900 $\left({ }^{2} \mathrm{E}_{\mathrm{g}} \rightarrow{ }^{2} \mathrm{~T}_{2 \mathrm{~g}}\right)$ in the electronic spectra suggest an octahedral geometry.[35] The $\mathrm{Zn}(\mathrm{II})$ complexes are diamagnetic as expected for $d^{10}$ system. (Table 2)

\subsection{Thermogravimetric Analysis}

Thermogravimetric analyses (TGA) for the complexes were carried out from room temperature to $700{ }^{\circ} \mathrm{C}$. Coordinated waters are usually eliminated at higher temperatures than those of hydration[36,37] usually in the temperature range $100-350{ }^{\circ} \mathrm{C}$. The complexes may decompose in more than two steps with the formation of intermediates[38,39] calculated and estimated mass losses are comparable.

The TGA curves of all Mannich-base metal complexes $\left(\mathrm{C}_{1}-\mathrm{C}_{24}\right)$ have two stages of mass loss, at $102-227^{\circ} \mathrm{C}$ and at $227-595{ }^{\circ} \mathrm{C}$. Weight loss in the range $102-227{ }^{\circ} \mathrm{C}$ with estimated mass loss of $3.72-4.85 \%$ in all the complexes indicates the loss of two coordinated waters. From $227^{\circ} \mathrm{C}$ to $595^{\circ} \mathrm{C}$, a sharp decrease in weight indicated the loss of one Mannich base from the complexes with estimated mass loss of $43.17-45.11 \%$ for all the complexes, respectively. The data are given in Table 3 .

The molecular masses determined mass spectrometrically also confirmed the $\mathrm{ML}_{2}$ composition. Based upon experimental evidence thus obtained, the complexes were characterized as six coordinates with the two positions occupied by water. The hydrated complexes have significant importance in the enzymatic systems, as the substrates can bind to metal by substituting the coordinated water. The proposed structures of the complexes under investigation, on the basis of above experimental evidence, are shown in Figure 1. Unsuccessful attempts to isolate crystals suitable for X-ray analysis prevented further structure elucidation.

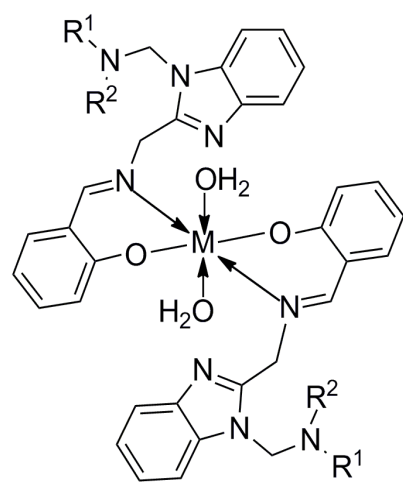

Where;

$\mathrm{M}=\mathrm{Co}(\mathrm{II}), \mathrm{Ni}(\mathrm{II}), \mathrm{Cu}(\mathrm{II}), \mathrm{Zn}(\mathrm{II})$

\subsection{Antibacterial Activity}

The in vitro antibacterial activity was assayed against two Gram-positive (Bacillus subtilis Staphylococcus aureus) and two Gram-negative strains (Escherichia coli, Pseudomonas aeruginosa) according to the reported method.[22] Gentamycine was used as a comparative drug.

The antibacterial results suggested that all the Mannich and Schiff base derivatives of benzimidazole were found to be biologically active. Aromatic amines seem to be more beneficial than aliphatic amines; $\mathrm{L}_{3}, \mathrm{~L}_{4}, \mathrm{~L}_{5}$ and $\mathrm{L}_{6}$ displayed the highest rate of suppression. Mannich bases $\mathrm{L}_{3}$ and $\mathrm{L}_{4}$ expressed almost the same activity as $\mathrm{L}_{4}$ has one additional Nitrogen atom in the ring, but $\mathrm{L}_{6}$ was described being the most antibacterial active molecule in this series.

In vitro efficiency of all the compounds against Gram-positive bacterial strains was much lower than Gram-negative. E. coli was the most susceptible species, affected by all the compounds. The activity against $S$. aureus is only mild even at $100 \mu \mathrm{gml}^{-1}$ concentration.

It is known[40,41] that chelation tends to make the Schiff bases act as more potent antibacterial agents. It is observed that growth inhibiting activity of metal(II) complexes of Mannich and Schiff bases is superior when compared with the ligands $\left(\mathrm{L}_{1}-\mathrm{L}_{6}\right.$ vs. $\left.\mathrm{C}_{1}-\mathrm{C}_{24}\right)$.

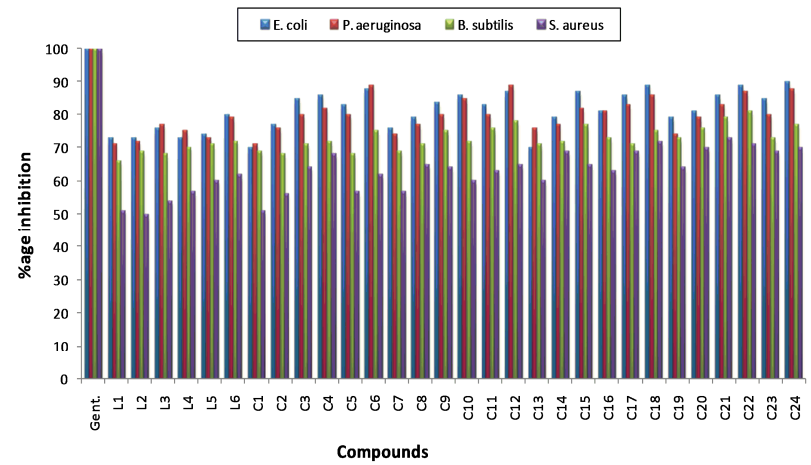

Figure 2. In vitro antibacterial spectrum of Mannich bases $\left(L_{1}-L_{6}\right)$ and $\mathrm{Zn}(I I), \mathrm{Co}(I I), \mathrm{Cu}(\mathrm{II}), \mathrm{Ni}(\mathrm{II})$ complexes $\left(\mathrm{C}_{1}-\mathrm{C}_{24}\right)$ and Gentamycine (Std.) at $100 \mu \mathrm{gml}^{-1}$ concentration.

\subsection{Antifungal Activity}

Antifungal activity was determined in vitro against Aspergillus niger, Penicillium expansum, Rhizopus nigricans, Trichoderna lignorum and Botrydepladia thiobromine. The inhibition results were compared with the standard drug Fluconazole.

Mannich and Schiff bases expressed lower antifungal activity as compared to antibacterial. All the derivatives were efficacious against $A$. niger and $P$. expansum. The results for $R$. nigricans and $T$. lignorum were satisfactory only by a high concentration, showing zone of inhibition in $60-70 \%$ range. $B$. thiobromine was almost insusceptible for all the Mannich and Schiff bases, but showed moderate results for complexes at higher concentration.

Figure 1. Proposed structure of Metal complexes. 


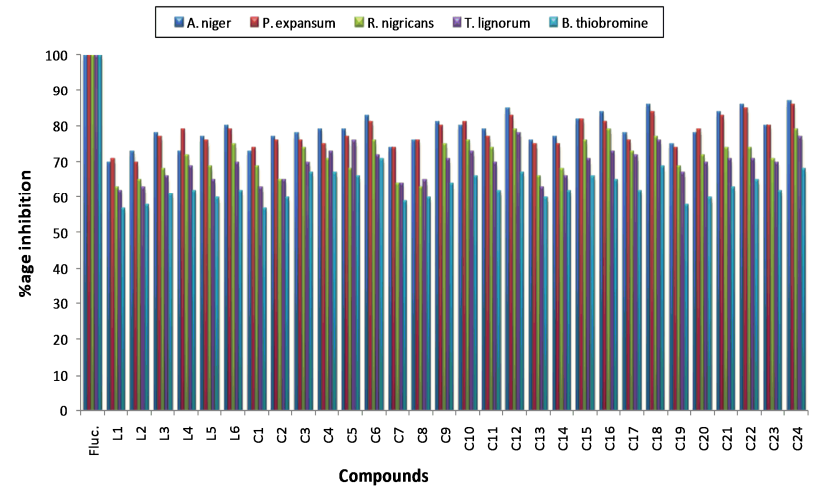

Figure 3. In vitro antifungal spectrum of Mannich bases $\left(L_{1}-L_{6}\right)$ and $Z n(I I)$, $\mathrm{Co}(I I), \mathrm{Cu}(I I), \mathrm{Ni}(I I)$ complexes $\left(\mathrm{C}_{1}-\mathrm{C}_{24}\right)$ and Fluconazole (Std.) at 100 $\mu \mathrm{gml}^{-1}$ concentration.

\subsection{Cytotoxic Bioassay}

Cytotoxicity (brine shrimp bioassay) was determined for all the compounds and their metal(II) complexes. The cytotoxicity is expressed as $\mathrm{LD}_{50}$, i.e. concentration, at which $50 \%$ of the viable cells were killed under the assay conditions.

From the data recorded in Table 5, it is evident that only one Mannich and Schiff base $\left(\mathrm{L}_{2}\right)$ displayed potent cytotoxic activity $\left(\mathrm{LD}_{50}=1.03 \times 10^{-4} \mathrm{moles} / \mathrm{mL}\right)$ against Artemia Salina, while the other synthesized compounds were almost inactive in this assay. It was interesting to note that complexation with copper increased cytotoxicity $\left(\mathrm{L}_{2}\right.$ vs. $\mathrm{C}_{14}$, all $\mathrm{Cu}(\mathrm{II})$ complexes), all other metal(II) complexes showed clearly higher values $\left(\mathrm{LD}_{50}>1.13 \mathrm{x} 10^{-3}\right.$ moles $/ \mathrm{mL}$ ). These findings may help to serve as a basis for future direction towards the development of bacteriostatic agents of lower cytotoxicity.

Table 4. Physical and Analytical data of Zn(II), Co(II), $\mathrm{Cu}(I I)$ and Ni(II) complexes

\begin{tabular}{|c|c|c|c|c|c|c|c|}
\hline \multirow{2}{*}{ Code } & \multirow{2}{*}{ Complex (formula weight) } & \multirow{2}{*}{ Yield (\%) } & \multirow{2}{*}{ Molar conductance $\left(\mathrm{Ohm}^{-1} \mathrm{~cm}^{-2} \mathrm{~mol}^{-1}\right)$} & \multicolumn{4}{|c|}{ Calculated (Found) \% } \\
\hline & & & & $\mathrm{C}$ & $\mathrm{H}$ & $\mathrm{N}$ & M \\
\hline $\mathrm{C}_{1}$ & {$\left[\mathrm{Zn}\left(\mathrm{L}_{1}\right)_{2}\left(\mathrm{OH}_{2}\right)_{2}\right](774.26)$} & 72 & 13.1 & $62.05(62.06)$ & $6.76(6.81)$ & $14.47(14.52)$ & $8.44(8.47)$ \\
\hline $\mathrm{C}_{2}$ & {$\left[\mathrm{Zn}\left(\mathrm{L}_{2}\right)_{2}\left(\mathrm{OH}_{2}\right)_{2}\right](748.18)$} & 71 & 14.2 & $57.79(57.81)$ & $6.19(6.21)$ & $18.72(18.73)$ & $8.74(8.77)$ \\
\hline $\mathrm{C}_{3}$ & {$\left[\mathrm{Zn}\left(\mathrm{L}_{3}\right)_{2}\left(\mathrm{OH}_{2}\right)_{2}\right](816.20)$} & 68 & 10.7 & $61.80(61.85)$ & $5.18(5.19)$ & $17.16(17.23)$ & $8.01(8.12)$ \\
\hline $\mathrm{C}_{4}$ & {$\left[\mathrm{Zn}\left(\mathrm{L}_{4}\right)_{2}\left(\mathrm{OH}_{2}\right)_{2}\right](818.18)$} & 72 & 9.6 & $58.71(58.77)$ & $4.92(4.96)$ & $20.54(20.58)$ & $7.99(8.05)$ \\
\hline $\mathrm{C}_{5}$ & {$\left[\mathrm{Zn}\left(\mathrm{L}_{5}\right)_{2}\left(\mathrm{OH}_{2}\right)_{2}\right](842.28)$} & 70 & 11.5 & $65.59(65.62)$ & $5.74(5.79)$ & $13.30(13.31)$ & $7.76(7.81)$ \\
\hline $\mathrm{C}_{6}$ & {$\left[\mathrm{Zn}\left(\mathrm{L}_{6}\right)_{2}\left(\mathrm{OH}_{2}\right)_{2}\right](966.42)$} & 72 & 8.6 & $69.63(69.68)$ & $5.42(5.43)$ & $11.59(11.62)$ & $6.76(6.79)$ \\
\hline $\mathrm{C}_{7}$ & {$\left[\mathrm{Co}\left(\mathrm{L}_{1}\right)_{2}\left(\mathrm{OH}_{2}\right)_{2}\right](767.79)$} & 76 & 7.8 & $65.57(65.59)$ & $6.82(6.83)$ & $14.59(14.61)$ & $7.67(7.79)$ \\
\hline $\mathrm{C}_{8}$ & {$\left[\mathrm{Co}\left(\mathrm{L}_{2}\right)_{2}\left(\mathrm{OH}_{2}\right)_{2}\right](741.71)$} & 72 & 6.9 & $58.29(58.37)$ & $6.25(6.26)$ & $18.88(18.91)$ & $7.94(7.97)$ \\
\hline $\mathrm{C}_{9}$ & {$\left[\mathrm{Co}\left(\mathrm{L}_{3}\right)_{2}\left(\mathrm{OH}_{2}\right)_{2}\right](809.73)$} & 70 & 5.4 & $62.29(62.37)$ & $5.22(5.26)$ & $17.29(17.31)$ & $7.27(7.31)$ \\
\hline $\mathrm{C}_{10}$ & {$\left[\mathrm{Co}\left(\mathrm{L}_{4}\right)_{2}\left(\mathrm{OH}_{2}\right)_{2}\right](811.71)$} & 73 & 6.1 & $59.18(59.19)$ & $4.96(4.99)$ & $20.70(20.71)$ & $7.25(7.27)$ \\
\hline $\mathrm{C}_{11}$ & {$\left[\mathrm{Co}\left(\mathrm{L}_{5}\right)_{2}\left(\mathrm{OH}_{2}\right)_{2}\right](835.81)$} & 71 & 9.3 & $66.10(66.17)$ & $5.78(5.78)$ & $13.40(13.41)$ & $7.05(7.11)$ \\
\hline $\mathrm{C}_{12}$ & {$\left[\mathrm{Co}\left(\mathrm{L}_{6}\right)_{2}\left(\mathrm{OH}_{2}\right)_{2}\right](959.95)$} & 69 & 4.9 & $70.06(70.10)$ & $5.46(5.49)$ & $11.67(11.73)$ & $6.13(6.19)$ \\
\hline $\mathrm{C}_{13}$ & {$\left[\mathrm{Cu}\left(\mathrm{L}_{1}\right)_{2}\left(\mathrm{OH}_{2}\right)_{2}\right](771.54)$} & 75 & 7.2 & $62.26(62.31)$ & $6.79(6.81)$ & $14.52(14.63)$ & $8.23(8.27)$ \\
\hline $\mathrm{C}_{14}$ & {$\left[\mathrm{Cu}\left(\mathrm{L}_{2}\right)_{2}\left(\mathrm{OH}_{2}\right)_{2}\right](746.32)$} & 72 & 7.9 & $57.93(57.94)$ & $6.21(6.28)$ & $18.76(18.77)$ & $8.51(8.53)$ \\
\hline $\mathrm{C}_{15}$ & {$\left[\mathrm{Cu}\left(\mathrm{L}_{3}\right)_{2}\left(\mathrm{OH}_{2}\right)_{2}\right](813.54)$} & 76 & 4.3 & $62.00(62.02)$ & $5.20(5.28)$ & $17.21(17.26)$ & $7.81(7.81)$ \\
\hline $\mathrm{C}_{16}$ & {$\left[\mathrm{Cu}\left(\mathrm{L}_{4}\right)_{2}\left(\mathrm{OH}_{2}\right)_{2}\right](816.32)$} & 68 & 11.4 & $58.85(58.87)$ & $4.93(4.97)$ & $20.59(20.65)$ & $7.78(7.79)$ \\
\hline $\mathrm{C}_{17}$ & {$\left[\mathrm{Cu}\left(\mathrm{L}_{5}\right)_{2}\left(\mathrm{OH}_{2}\right)_{2}\right](840.42)$} & 72 & 12.6 & $65.74(65.81)$ & $5.75(5.81)$ & $13.33(13.47)$ & $7.56(7.59)$ \\
\hline $\mathrm{C}_{18}$ & {$\left[\mathrm{Cu}\left(\mathrm{L}_{6}\right)_{2}\left(\mathrm{OH}_{2}\right)_{2}\right](964.54)$} & 71 & 8.7 & $69.73(69.81)$ & $5.43(5.47)$ & $11.61(11.63)$ & $6.58(6.62)$ \\
\hline $\mathrm{C}_{19}$ & {$\left[\mathrm{Ni}\left(\mathrm{L}_{1}\right)_{2}\left(\mathrm{OH}_{2}\right)_{2}\right](767.55)$} & 74 & 13.6 & $62.59(62.61)$ & $6.82(6.83)$ & $14.59(14.63)$ & $7.64(7.71)$ \\
\hline $\mathrm{C}_{20}$ & {$\left[\mathrm{Ni}\left(\mathrm{L}_{2}\right)_{2}\left(\mathrm{OH}_{2}\right)_{2}\right](741.47)$} & 76 & 7.9 & $58.34(58.41)$ & $6.25(6.37)$ & $18.89(18.89)$ & $7.91(7.96)$ \\
\hline $\mathrm{C}_{21}$ & {$\left[\mathrm{Ni}\left(\mathrm{L}_{3}\right)_{2}\left(\mathrm{OH}_{2}\right)_{2}\right](809.49)$} & 70 & 12.6 & $62.31(62.32)$ & $5.22(5.22)$ & $17.30(17.31)$ & $7.25(7.27)$ \\
\hline $\mathrm{C}_{22}$ & {$\left[\mathrm{Ni}\left(\mathrm{L}_{4}\right)_{2}\left(\mathrm{OH}_{2}\right)_{2}\right](811.47)$} & 72 & 11.3 & $59.20(59.22)$ & $4.96(4.99)$ & $20.71(20.73)$ & $7.23(7.27)$ \\
\hline $\mathrm{C}_{23}$ & {$\left[\mathrm{Ni}\left(\mathrm{L}_{5}\right)_{2}\left(\mathrm{OH}_{2}\right)_{2}\right](835.54)$} & 72 & 9.7 & $66.12(66.16)$ & $5.79(5.79)$ & $13.41(13.42)$ & $7.02(7.02)$ \\
\hline $\mathrm{C}_{24}$ & {$\left[\mathrm{Ni}\left(\mathrm{L}_{6}\right)_{2}\left(\mathrm{OH}_{2}\right)_{2}\right](959.71)$} & 69 & 12.5 & $70.08(70.08)$ & $5.46(5.47)$ & $11.67(11.68)$ & $6.11(6.12)$ \\
\hline
\end{tabular}


Table 5. Brine shrimp bioassay data of the Mannich bases $\left(L_{1}-L_{6}\right)$ and their metal(II) complexes $\left(C_{1}-C_{24}\right)$

\begin{tabular}{cccclc}
\hline Code & $\mathbf{L D}_{\mathbf{5 0}}(\mathbf{M})$ & $\mathrm{Code}$ & $\mathbf{L D}_{\mathbf{5 0}}(\mathbf{M})$ & Code & $\mathbf{L D}_{\mathbf{5 0}}(\mathbf{M})$ \\
\hline $\mathrm{L}_{1}$ & $>3.36 \times 10^{-3}$ & $\mathrm{C}_{5}$ & $>1.59 \times 10^{-3}$ & $\mathrm{C}_{15}$ & $1.67 \times 10^{-4}$ \\
$\mathrm{~L}_{2}$ & $1.03 \times 10^{-4}$ & $\mathrm{C}_{6}$ & $>1.44 \times 10^{-3}$ & $\mathrm{C}_{16}$ & $2.87 \times 10^{-4}$ \\
$\mathrm{~L}_{3}$ & $>2.94 \times 10^{-3}$ & $\mathrm{C}_{7}$ & $>1.13 \times 10^{-3}$ & $\mathrm{C}_{17}$ & $1.89 \times 10^{-4}$ \\
$\mathrm{~L}_{4}$ & $>2.87 \times 10^{-3}$ & $\mathrm{C}_{8}$ & $>1.27 \times 10^{-3}$ & $\mathrm{C}_{18}$ & $3.74 \times 10^{-4}$ \\
$\mathrm{~L}_{5}$ & $>2.61 \times 10^{-3}$ & $\mathrm{C}_{9}$ & $>1.69 \times 10^{-3}$ & $\mathrm{C}_{19}$ & $>1.41 \times 10^{-3}$ \\
$\mathrm{~L}_{6}$ & $>2.02 \times 10^{-3}$ & $\mathrm{C}_{10}$ & $>1.65 \times 10^{-3}$ & $\mathrm{C}_{20}$ & $2.70 \times 10^{-4}$ \\
$\mathrm{C}_{1}$ & $>1.30 \times 10^{-3}$ & $\mathrm{C}_{11}$ & $>1.87 \times 10^{-3}$ & $\mathrm{C}_{21}$ & $>1.73 \times 10^{-3}$ \\
$\mathrm{C}_{2}$ & $3.11 \times 10^{-4}$ & $\mathrm{C}_{12}$ & $>1.63 \times 10^{-3}$ & $\mathrm{C}_{22}$ & $>1.82 \times 10^{-3}$ \\
$\mathrm{C}_{3}$ & $>1.31 \times 10^{-3}$ & $\mathrm{C}_{13}$ & $1.01 \times 10^{-4}$ & $\mathrm{C}_{23}$ & $>1.61 \times 10^{-3}$ \\
$\mathrm{C}_{4}$ & $>1.82 \times 10^{-3}$ & $\mathrm{C}_{14}$ & $1.83 \times 10^{-5}$ & $\mathrm{C}_{24}$ & $>1.97 \times 10^{-3}$ \\
\hline
\end{tabular}

\section{Conclusion}

The synthesized Mannich and Schiff bases act as bidentate ligands. The IR, TGA, conductivity, magnetic and electronic studies confirm that the metals are coordinated to azomethine nitrogen and phenolic oxygen. All the derivatives and their metal(II) complexes were evaluated in vitro against four bacterial (two Gram-negative, two Gram-positive) and five fungal strains. Compounds showed more potency against bacteria. E. coli, P. aeruginosa, A. niger and $P$. expansum were the most susceptible species.

\section{Acknowledgements}

The author thanks to the Higher Education Commission (HEC), Government of Pakistan for awarding Indigenous Scholarship and supporting research facilities. We also thank to QAU, Islamabad for providing spectroscopic services. Finally, HEJ Research Institute of Chemistry, University of Karachi is also acknowledged for undertaking the biological assays.

\section{References}

[1] X. C. Huang, J. P. Zhang, X. M. Chen, J. Am. Chem. Soc. 126, 2004, 13218-13219.

[2] T. J. Cardwell, A. J. Edwards, R. M. Hartshorn, R. J. Holmes, Aust. J. Chem. 50, 1997, 1009-1015.

[3] Y. P. Tong and S. L. Zheng, J. Mol. Struct. 841, 2007, 34-40.

[4] Y. P. Tong, S.L. Zheng, and X.M. Chen, J. Mol. Struct. 826, 2007, 104-112.

[5] S. Murugesan, S. Sathiyamoorthy, J. Pharm. Res. 4, 2011, 2679-2681.

[6] V. Kamlesh, Patel, and S. Arun, Eur. J. Chem. 6, 2009, 281-288.

[7] B.A. Reddy, Eur. J. Chem. 7, 2010, 222-226.
[8] S. Periyasamy, R.L. Dhani, P. Christophe, Int. J. Pharmaceut. Sci. Res. 1, 2010, 105-109.

[9] E.P. Jesudason, S.K. Sridhar, E.J. Padma, et al., Eur. J. Med. Chem. 44, 2009, 2307-2312.

[10] [M. Arif, M.M.R. Qurashi, M.A. Shad, J. Coord. Chem. 64:11, 2011, 1914-1930.

[11] J. Pragathi, K. Mounika, M. Padmaja, M. Lakshmi, C. Gyanakumari, Eur. J. Chem. 8, 2011, 1662-1669.

[12] Y.S. Chhonker, B. Veenu, S.R. Hasim, et al., Eur. J. Chem. 6, 2009, S342-S346.

[13] H. Marijana, S. Kristina, K. Sandra, et al., Eur. J. Med. Chem. 46, 2011, 2274-2279.

[14] [K.C. Rout, R.R. Mohanty, S. Jena, K.C. Dash, Polyhedron $15,1996,1023$

[15] H. Chang, M. Fu, X.J. Zhao, E.C. Yang, J. Coord. Chem. 63, $2010,3551$.

[16] F.M. Nie, J. Chen, Z. Li, F. Lu, J. Coord. Chem. 63, 2010, 1711.

[17] M.Y. Duan, J. Li, Y. Xi, X.F. Lu, J.Z. Liu, G. Mele, F.X. Zhang, J. Coord. Chem. 63, 2010, 90.

[18] M. Akkurt, S. Karaca, H. Kucukbay, E. Orhan, O. Buyukgungor, Acta Cryst. 2005, E61, m41.

[19] H. Kucukbay, S. Gunal, E. Orhan, R. Durmaz. Asian J. Chem. $22,2010,7376$

[20] R.M. Mannar, K. Amit, E. Martin, R. Dieter, Inorg. Chem. 45, 2006, 5924-5937.

[21] Atta-ur-Rahman, M.I. Choudhary, W.J. Thomsen, Bioassay techniques for drug development, Harwood Academic Publishers, The Netherlands, 2001.

[22] K.S. Anil, M. Yasmin, R.A. Kamal, P. Om, Eur. J. Med. Chem. 38, 2003, 533-536.

[23] Atta-ur-Rahman, M.I. Choudhary, W.J. Thomsen, Bioassay techniques for drug development, Harwood Academic Publishers, The Netherlands, 2001. 
[24] B. N. Meyer, N. R. Ferrigni, J. E. Putnam, et. al., Planta Med. 45, 1982, 31-34.

[25] A.W. Bauer, W.M. Kirby, J.C. Sherris, M. Turck, Am. J. Clin. Pathol. 45, 1966, 493-496.

[26] K. Nakamoto, Infrared and raman spectra of inorganic and coordination compounds, 5th edn. John Wiley \& Sons Inc., New York, 1997.

[27] J.R. Ferrero, Low-frequency Vibrations of Inorganic and Coordination Compounds. Wiley-Interscience, New York, 1971.

[28] G. Singh, P.A. Singh, K. Singh, et. al., Proc. Nat. Acad. Sci. Ind. $72 \mathrm{~A}, 2002,87-94$.

[29] R. Sayaji, Asian J. Chem. 17, 2005, 2663-2668.

[30] W.W. Simons, The Sadtler handbook of proton NMR spectra, Sadtler Research Laboratories Inc. Philadelphia, 1978.

[31] D.J. Pasto, Organic structure determination, Prentice Hall International, London, 1969.

[32] S. Murugesan, S. Sathiyamoorthy, J. Pharm. Res. 4, 2011, $2679-2681$
[33] R. K. Agarwal, P. Garg, H. Agarwal, S. Chandra, Synth. React. Inorg. Met.-Org. Chem. 27, 1997, 251.

[34] P. K. Panchal, M. N. Patel, Synth. React. Inorg. Met.-Org. Chem. 34, 2004, 1277-1289.

[35] H. Koksal, M. Dolaz, M. Tilmer and S. Serin, Synth. React. Inorg. Met.-Org. Chem. 31, 2001, 1141-1162.

[36] G.G. Mohamed, Z.H. Abd El-Wahab. J. Therm. Anal. Calorim. 73, 2003, 347.

[37] Y.M. Essa, H.M. Abdel-Fattah, A.A. Soliman. J. Therm. Anal. 42, 1994, 1175.

[38] N.T. Abdel-Ghani, O. Esherif. Thermochim. Acta. 156, 1989, 69.

[39] M.A. Zayed, F.A. Nour El-Dien, G.G. Mohamed, N.E.A. El-Gamel. Spectrochim. Acta Part A, 60, 2004, 2843.

[40] Z.H. Chohan, C.T. Supuran, A. Scozzafava, J. Enzyme Inhib. Med. Chem. 19, 2004, 79-84.

[41] Z.H. Chohan, M. Praveen, Appl. Organomet. Chem. 15, 2001, 617-625. 